\title{
Dynamic fracture analysis of finite cracks by horizontally polarized shear waves in anisotropic solids
}

\author{
Yi-Shyong Ing ${ }^{\mathrm{a}}$, Chien-Ching $\mathrm{Ma}^{\mathrm{b}, *}$ \\ a Department of Aerospace Engineering, Tamkang University, Tamsui, Taiwan 251, ROC \\ ${ }^{\mathrm{b}}$ Department of Mechanical Engineering, National Taiwan University, 1 Roosevelt Road, Sec. 4, \\ Taipei, Taiwan 106, ROC
}

\begin{abstract}
In this study, the dynamic fracture analysis of finite cracks in anisotropic elastic solids subjected to incident horizontally polarized shear waves is investigated. The influence of finite length of the crack on the dynamic stress intensity factor will be discussed in detail. A linear coordinate transformation is introduced to simplify the problem. The linear coordinate transformation reduces the anisotropic finite crack problem to an equivalent isotropic problem. An alternative methodology different from the conventional superposition method is developed to construct the diffracted fields. The transient solutions are determined by superposition of two proposed fundamental solutions in the Laplace transform domain. For stationary cracks, the exact analytical solutions of dynamic stress intensity factors for two crack tips are obtained in explicit forms and have accounted for the contributions of all the diffracted waves. For a step-stress wave, the maximum dynamic overshot of stress intensity factor is $4 / \pi$ for any combination of material constants and incident angles. If the stress intensity factor reaches the fracture toughness of the material, the two crack tips are assumed to propagate along the crack tip line with constant subsonic velocities. The influence of the diffracted waves generated from the other crack tip on the propagating crack tip is analyzed. It is shown in this study that the diffracted waves from the other crack tip have significant influence on the stress intensity factors for propagating cracks. (C) 2003 Elsevier Ltd. All rights reserved.
\end{abstract}

Keywords: Finite cracks; Anisotropic materials; Propagating cracks; Dynamic fracture; Transient waves

\section{Introduction}

Scattering of elastic waves by cracks has aroused attention over the years for its importance toward the non-destructive evaluation of materials and the dynamic fracture

\footnotetext{
* Corresponding author. Tel.: +886-2-2365-9996; fax: +886-2-2363-1755.

E-mail address: ccma@ntu.edu.tw (C.-C. Ma).
} 
analysis of materials. The interaction of a stress wave with a crack is a complicated problem and the analysis is mainly restricted to relatively simple problems. Most of the works for dynamic fracture analysis have been directed toward the solutions of problems without any characteristic length. The complete solutions of this kind of problems can be obtained by integral transform methods in conjunction with direct application of the Wiener-Hopf technique (Noble, 1958) and the Cagniard-de Hoop method (de Hoop, 1958) of Laplace inversion. If the cracked problem has a characteristic length or the loading condition is unsymmetrical, then it is hard to apply the same procedure using integral transform methods. The problem of an unbounded elastic solid containing a semi-infinite crack subjected to a pair of concentrated point loadings on the crack faces has been studied by Freund (1974a). A straightforward application of the Wiener-Hopf method was not successful and he proposed a fundamental solution arising from an edge dislocation climbing along the line ahead of the crack tip with a constant speed to overcome the difficulties of the case with a characteristic length. The solution can be constructed by taking integration over a climbing edge dislocation of different moving velocity. The limitation of above-mentioned problems is that the incident field must be represented as a function of $f(t / x)$, say self-similar. For problems of non-infinite domain or with complicated loading conditions, the solutions cannot be obtained by using this method. A powerful and efficient methodology based on superimposing a fundamental solution in the Laplace transform domain was proposed by Tsai and Ma (1992). Exact transient closed form solutions of stresses and dynamic stress intensity factors for a stationary semi-infinite crack subjected to a suddenly applied dynamic body force in an unbounded medium have been obtained by Tsai and Ma (1992) for the in-plane case. This superposition methodology was generalized and applied to analyze the propagating crack interacting with boundaries for in-plane deformation by Tsai and Ma (1997a,b), but only the effects of first few reflected waves from the boundaries were taken into account.

Many researchers have already devoted to the study of finite-crack problems for isotropic materials. Thau and $\mathrm{Lu}$ (1971), following the work of Kostrov (1964) and Flitman (1963), treated the transient problem of diffraction of an arbitrary plane dilatational wave by a finite crack in an infinite elastic solid. However, their results are exact only at the time interval that the dilatational wave has traveled the length of the crack twice. Sih and Embley (1972) have studied the near-field solution for the problem of a finite crack under transient in-plane loading. They reduced the mixed boundary value problem to a standard Fredholm integral equation and subsequently inverted the Laplace transform of the stress components by a combination of numerical means and an application of the Cagniard inversion technique. Most of the investigators, however, have either solved problems of a finite crack valid for a short time, or finally used a numerical technique to obtain the solutions in the physical domain. Because of the mathematical difficulties, the transient closed form analytical solution for the problem of a finite crack has not been attempted until Ing and Ma $(1996,1997)$ proposed useful fundamental solutions to overcome this difficulty.

Finite cracks in orthotropic materials under dynamic loadings have been investigated by Kassir and Bandyopadhyay (1983), Shindo and Nozaki (1991), Rubio-Gonzalez and Mason $(1999,2001)$ using integral transform method. This method leads to a 
Fredholm integral equation rather than a Wiener-Hopf equation on the Laplace transform domain. They solved the Fredholm integral equation in the Laplace transform domain numerically, and the dynamic stress intensity factor was obtained by numerical Laplace inversion. Zhang (2000) applied the time-domain traction boundary integral equation (BIE) to obtain the dynamic stress intensity factor of a finite crack in anisotropic solids under anti-plane deformation. Albuquerque et al. (2002) developed a multi-domain boundary element formulation to solve a two-dimensional dynamic anisotropic problem with cracks. They computed the dynamic stress intensity factors by using traction singular quarter point elements.

In the study of crack propagation, Yoffe (1951) began to investigate a steady-state crack growth problem of a crack of fixed length propagating in an infinite elastic body subjected to a uniform remote tensile stress. Kostrov (1966) and Achenbach (1970a,b) have used the method based on Green's function to solve the problems of crack propagation for anti-plane deformation. In a series of paper, Freund (1972a,b, 1973, 1974b) developed important analytical methods for evaluation of the transient stress field of a propagating crack in a homogeneous material under quite general dynamic loading situation. An indirect analytical approach was proposed by Freund based on superposition over fundamental solutions. Based on the superposition method proposed by Freund, a series of problems for non-planar crack propagation in an infinite domain were solved by Ma and Burgers (1986, 1987, 1988) and Ma (1988, 1990). For the aforementioned problems, either the direct application of the well-known Wiener-Hopf technique (Noble, 1958) is used or the superposition method proposed by Freund is performed to solve the problem. However, if a crack is subjected to incident non-planar waves, it is hard to use the well-known methods directly to obtain the transient solutions. The major reference on the dynamic fracture mechanics can be found in the extensive monograph by Freund (1990). Recently, Rosakis (2002) reviewed the dynamic crack propagation of intersonic shear rupture of theoretical and experimental results. Guo et al. (2003) investigated the problem of rapid shear cracking in isotropic materials by numerical simulation and experiments. They obtained analytical solutions for a sub-Rayleigh or an intersonic crack by either accelerating or decelerating to a different cracking speed. Samudrala and Rosakis (2003) studied the nature of intersonic crack propagation along a bimaterial interface by optical techniques.

Because of the mathematical difficulties, the explicitly analytical solution for the dynamic fracture problem of a propagating finite crack subjected to dynamic loading in anisotropic solids has not yet been obtained. In this study, the theoretical transient analysis is performed for finite cracks in unbounded anisotropic media subjected to horizontally polarized shear waves as shown in Fig. 1. Investigations on the problems for anisotropic materials are tedious due to the presence of many material constants. It is desirable to reduce the dependence on material constants in advance of the analysis of a given problem. A linear coordinate transformation is introduced in this study to simplify the problems. Based on this transformation, the original problems of anisotropic materials with finite cracks are converted to equivalent isotopic problems with a similar geometrical configuration. In analyzing this problem, the interaction of waves with two crack tips must be taken into account and it is impossible to solve this complicated problem by using the standard integral transform method. Two useful 


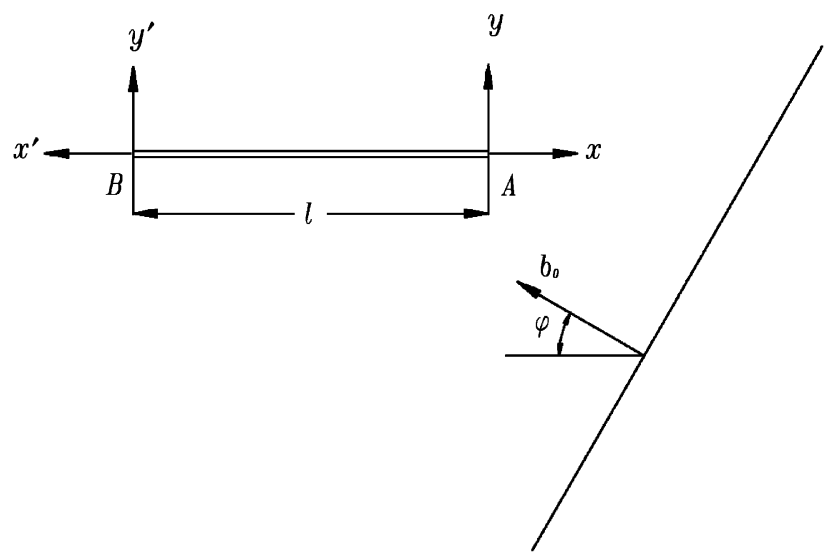

Fig. 1. Schematic and coordinate systems of a finite crack subjected to an incident horizontally polarized shear wave.

fundamental solutions are proposed to overcome these difficulties. The exact solutions of dynamic stress intensity factors are derived for the anisotropic stationary finite crack problem. Based on the analytical solutions, the transient responses of stress intensity factors are discussed in detail by numerical calculations. The analytical solutions show that the dynamic stress intensity factor of a stationary finite crack will reach a maximum value, and then decrease and oscillate near the static value. If the dynamic stress intensity factor reaches the fracture toughness of the material, then the two crack tips of the finite crack are assumed to propagate with constant subsonic velocities along the crack tip line. The influence of the first two diffracted waves generated from the other crack tip on the dynamic stress intensity factor of the propagating crack is analyzed and discussed in detail.

\section{A linear coordinate transformation and fundamental solutions}

The anti-plane anisotropic problem can be converted to a corresponding isotropic problem by properly changing the geometry of the original configuration and the tractions on the boundary. In other words, the anisotropic anti-plane problem can be simplified to an isotropic problem with the aid of a suitable coordinate transformation. By using a linear coordinate transformation, the complete full-field static solutions of anisotropic multilayered media subjected to concentrated shear forces and screw dislocations in an arbitrary layer were obtained by Lin and Ma (2000).

In this section, a linear coordinate transformation and two fundamental problems are proposed to solve the problem of a finite crack in an anisotropic material subjected to horizontally polarized shear waves and propagates with constant velocities. The solutions for an exponentially distributed traction applied on the propagating crack faces and an exponentially distributed displacement along the propagating crack tip line in the Laplace transform domain will be referred to as the fundamental solutions. 
The diffracted waves generated from two crack tips can be successfully constructed by superimposing the fundamental solutions in the Laplace transform domain.

\subsection{The linear coordinate transformation}

Consider the problem of a semi-infinite crack propagating in an anisotropic unbounded medium under anti-plane deformation. The crack is assumed to propagate along the crack line (i.e. positive $x$-axis) with a constant subsonic velocity $v$. In analyzing this problem, it is convenient to express the governing equations of wave motions in the moving coordinate $(\xi, y)$ which is attached to the propagating crack tip. For the absence of body force, the two-dimensional wave motion of a homogeneous anisotropic solid under anti-plane deformation in terms of the displacement is governed by

$$
\begin{aligned}
& C_{55} \frac{\partial^{2} w(\xi, y, t)}{\partial \xi^{2}}+2 C_{45} \frac{\partial^{2} w(\xi, y, t)}{\partial \xi \partial y}+C_{44} \frac{\partial^{2} w(\xi, y, t)}{\partial y^{2}} \\
&=\rho\left(v^{2} \frac{\partial^{2} w(\xi, y, t)}{\partial \xi^{2}}-2 v \frac{\partial^{2} w(\xi, y, t)}{\partial \xi \partial t}+\frac{\partial^{2} w(\xi, y, t)}{\partial t^{2}}\right),
\end{aligned}
$$

where $\xi=x-v t, w(\xi, y, t)$ is the out-of-plane displacement in the $z$-direction, $C_{i j}(i, j=$ $4,5)$ are elastic moduli and $\rho$ is the mass density of the anisotropic material. The $\xi y$-plane has been assumed to coincide with one of the plane of material symmetry so that in-plane and anti-plane deformations are uncoupled. The relevant shear stress components are

$$
\begin{aligned}
\tau_{y z}(\xi, y, t) & =C_{44} \frac{\partial w(\xi, y, t)}{\partial y}+C_{45} \frac{\partial w(\xi, y, t)}{\partial \xi}, \\
\tau_{\xi z}(\xi, y, t) & =C_{45} \frac{\partial w(\xi, y, t)}{\partial y}+C_{55} \frac{\partial w(\xi, y, t)}{\partial \xi} .
\end{aligned}
$$

Introduce a linear coordinate transformation (Ma, 1996; Lin and Ma, 2000)

$$
\begin{aligned}
& \varsigma=\xi-\frac{C_{45}}{C_{44}} y, \\
& Y=\frac{C_{e}}{C_{44}} y, \\
& Z=z,
\end{aligned}
$$

where

$$
C_{e}=\sqrt{C_{44} C_{55}-C_{45}^{2}} \text {. }
$$

Assume that $C_{44}$ and $C_{45}$ as well as $\sqrt{C_{44} C_{55}-C_{45}^{2}}$ are all positive. The transformation given by Eqs. (4)-(6) reduces Eq. (1) to the standard wave equation for the 
isotropic solid in the $(\varsigma, Y)$ coordinate system as

$$
\left(1-b v^{2}\right) \frac{\partial^{2} W(\varsigma, Y, t)}{\partial \varsigma^{2}}+2 b^{2} v \frac{\partial^{2} W(\varsigma, Y, t)}{\partial \varsigma \partial t}+\frac{\partial^{2} W(\varsigma, Y, t)}{\partial Y^{2}}=b^{2} \frac{\partial^{2} W(\varsigma, Y, t)}{\partial t^{2}},
$$

where $W(\varsigma, Y, t)$ is the displacement in the $Z$-direction and

$$
\begin{aligned}
& W(\varsigma, Y, t)=w(\xi, y, t), \\
& b=\frac{\sqrt{C_{44} \rho}}{C_{e}} .
\end{aligned}
$$

It is easy to verify from Eqs. (2) and (3) that the relevant stress components in the anisotropic solid are related to those in the corresponding isotropic solid by

$$
\begin{aligned}
\tau_{Y Z}(\varsigma, Y, t) & =C_{e} \frac{\partial W(\varsigma, Y, t)}{\partial Y}, \\
\tau_{\varsigma Z}(\varsigma, Y, t) & =C_{e} \frac{\partial W(\varsigma, Y, t)}{\partial \varsigma}, \\
\tau_{y z}(\xi, y, t) & =\tau_{Y Z}(\varsigma, Y, t), \\
\tau_{\xi z}(\xi, y, t) & =\frac{C_{45}}{C_{44}} \tau_{Y Z}(\varsigma, Y, t)+\frac{C_{e}}{C_{44}} \tau_{\varsigma Z}(\varsigma, Y, t) .
\end{aligned}
$$

From Eqs. (8), (11) and (12), it is noted that the original anisotropic problem is converted into an equivalent isotropic problem by setting $C_{e}=\mu$ (shear modulus). From the relationship of displacement and shear stresses for an anisotropic solid and the correspondent isotropic solid expressed in Eqs. (9), (13) and (14), one can see that it is possible to obtain the solution for an anisotropic problem from a correspondent result of an isotropic problem.

\subsection{The fundamental solution of distributed loads applied on propagating crack faces}

Consider an anisotropic material in an unbounded medium containing a semi- infinite crack which lies on the negative $\xi$-axis and propagates with a constant velocity. An anti-plane exponentially distributed traction in the Laplace transform domain is applied on the upper and lower crack faces of the propagating crack. The boundary conditions expressed in the Laplace transform domain for this fundamental problem are represented as follows:

$$
\begin{aligned}
& \bar{\tau}_{y z}(\xi, 0, s)=\mathrm{e}^{s \eta \xi} \quad \text { for }-\infty<\xi<0, \\
& \bar{w}(\xi, 0, s)=0 \quad \text { for } 0<\xi<\infty,
\end{aligned}
$$

where $s$ is the Laplace transform parameter and $\eta$ is a constant. The overbar symbol is used for denoting the transform on time $t$. By using Eqs. (4)-(6), (9) and (13), the 
boundary conditions Eqs. (15) and (16) are rewritten as

$$
\begin{aligned}
& \bar{\tau}_{Y Z}(\varsigma, 0, s)=\mathrm{e}^{s \eta \varsigma} \quad \text { for }-\infty<\varsigma<0, \\
& \bar{W}(\varsigma, 0, s)=0 \quad \text { for } 0<\varsigma<\infty .
\end{aligned}
$$

The fundamental problem for the governing equation (8) subjected to boundary conditions Eqs. (17) and (18) can be solved by the application of standard integral transform methods. The one-sided Laplace transform with respect to time and the two-sided Laplace transform with respect to $\varsigma$ of a function are defined by (Achenbach, 1973)

$$
\begin{aligned}
& \bar{f}(\varsigma, Y, s)=\int_{0}^{\infty} f(\varsigma, Y, t) \mathrm{e}^{-s t} \mathrm{~d} t, \\
& \bar{f}^{*}(\lambda, Y, s)=\int_{-\infty}^{\infty} \bar{f}(\varsigma, Y, s) \mathrm{e}^{-s \lambda \varsigma} \mathrm{d} \varsigma .
\end{aligned}
$$

Apply the one-sided Laplace transform over time, and the two-sided Laplace transform over $\varsigma$ under the restriction of $\operatorname{Re}(\eta)>\operatorname{Re}(\lambda)$, and the Wiener-Hopf technique is finally implemented. The solutions of shear stresses and displacement for this fundamental problem in the Laplace transform domain can be expressed as follows:

$$
\begin{aligned}
& \bar{\tau}_{Y Z}(\varsigma, Y, s)=\frac{1}{2 \pi \mathrm{i}} \int_{\Gamma_{\lambda}} \frac{\alpha_{+}^{*}(\lambda) \mathrm{e}^{-s\left[\alpha^{*}(\lambda)|Y|-\lambda \varsigma\right]}}{\alpha_{+}^{*}(\eta)(\eta-\lambda)} \mathrm{d} \lambda, \\
& \bar{\tau}_{\varsigma Z}(\varsigma, Y, s)=\frac{-\operatorname{sign}(Y)}{2 \pi \mathrm{i}} \int_{\Gamma_{\lambda}} \frac{\lambda \mathrm{e}^{-s\left[\alpha^{*}(\lambda)|Y|-\lambda \varsigma\right]}}{\alpha_{+}^{*}(\eta)(\eta-\lambda) \alpha_{-}^{*}(\lambda)} \mathrm{d} \lambda, \\
& \bar{W}(\varsigma, Y, s)=\frac{-\operatorname{sign}(Y)}{2 \pi \mathrm{i}} \int_{\Gamma_{\lambda}} \frac{\mathrm{e}^{-s\left[\alpha^{*}(\lambda)|Y|-\lambda \varsigma\right]}}{C_{e} s \alpha_{+}^{*}(\eta)(\eta-\lambda) \alpha_{-}^{*}(\lambda)} \mathrm{d} \lambda,
\end{aligned}
$$

where $\Gamma_{\lambda}$ is the path of integration in the complex $\lambda$-plane and

$$
\begin{aligned}
& \operatorname{sign}(Y)= \begin{cases}1 & \text { if } Y \geqslant 0^{+}, \\
-1 & \text { if } Y \leqslant 0^{-}\end{cases} \\
& \alpha_{+}^{*}(\lambda)=\sqrt{b+\lambda(1-b v)}, \quad \alpha_{-}^{*}(\lambda)=\sqrt{b-\lambda(1+b v)}, \\
& \alpha^{*}(\lambda)=\alpha_{+}^{*}(\lambda) \alpha_{-}^{*}(\lambda) .
\end{aligned}
$$

The corresponding result of the dynamic stress intensity factor in the Laplace transform domain is

$$
\bar{K}(s)=\lim _{\varsigma \rightarrow 0^{+}} \sqrt{2 \pi \varsigma} \bar{\tau}_{Y Z}(\varsigma, 0, s)=-\frac{\sqrt{2(1-b v)}}{\sqrt{s} \alpha_{+}^{*}(\eta)} .
$$

If the propagating velocity of the crack $v=0$ which is correspondent to the stationary crack, then Eqs. (21)-(24) can be reduced to

$$
\bar{\tau}_{Y Z}(X, Y, s)=\frac{1}{2 \pi \mathrm{i}} \int_{\Gamma_{\lambda}} \frac{\alpha_{+}(\lambda) \mathrm{e}^{-s[\alpha(\lambda)|Y|-\lambda X]}}{\alpha_{+}(\eta)(\eta-\lambda)} \mathrm{d} \lambda,
$$




$$
\begin{aligned}
& \bar{\tau}_{X Z}(X, Y, s)=\frac{-\operatorname{sign}(Y)}{2 \pi \mathrm{i}} \int_{\Gamma_{\lambda}} \frac{\lambda \mathrm{e}^{-s[\alpha(\lambda)|Y|-\lambda X]}}{\alpha_{+}(\eta)(\eta-\lambda) \alpha_{-}(\lambda)} \mathrm{d} \lambda, \\
& \bar{W}(X, Y, s)=\frac{-\operatorname{sign}(Y)}{2 \pi \mathrm{i}} \int_{\Gamma_{\lambda}} \frac{\mathrm{e}^{-s[\alpha(\lambda)|Y|-\lambda X]}}{C_{e} s \alpha_{+}(\eta)(\eta-\lambda) \alpha_{-}(\lambda)} \mathrm{d} \lambda, \\
& \bar{K}(s)=\lim _{X \rightarrow 0^{+}} \sqrt{2 \pi X} \bar{\tau}_{Y Z}(X, 0, s)=-\frac{\sqrt{2}}{\sqrt{s} \alpha_{+}(\eta)},
\end{aligned}
$$

where $X=\varsigma+v t$ and

$$
\begin{aligned}
& \alpha_{+}(\lambda)=\sqrt{b+\lambda}, \quad \alpha_{-}(\lambda)=\sqrt{b-\lambda}, \\
& \alpha(\lambda)=\alpha_{+}(\lambda) \alpha_{-}(\lambda) .
\end{aligned}
$$

\subsection{The fundamental solution of distributed displacement ahead of a propagating} crack tip

Consider a semi-infinite propagating crack contained in an unbounded anisotropic medium. An exponentially distributed displacement ahead of the propagating crack tip yields the following boundary conditions in the Laplace transform domain:

$$
\begin{aligned}
& \bar{w}\left(\xi, 0^{+}, s\right)=\mathrm{e}^{s \eta \xi} \quad \text { for } 0<\xi<\infty, \\
& \bar{w}\left(\xi, 0^{-}, s\right)=-\mathrm{e}^{s \eta \xi} \quad \text { for } 0<\xi<\infty, \\
& \bar{\tau}_{y z}(\xi, 0, s)=0 \quad \text { for }-\infty<\xi<0 .
\end{aligned}
$$

Follow a similar procedure as mentioned previously. The solutions of stresses and displacement expressed in the Laplace transform domain are

$$
\begin{aligned}
& \bar{\tau}_{Y Z}(\varsigma, Y, s)=\frac{1}{2 \pi \mathrm{i}} \int_{\Gamma_{\lambda}} \frac{C_{e} s \alpha_{-}^{*}(\eta) \alpha_{+}^{*}(\lambda) \mathrm{e}^{-s[\alpha(\lambda)|Y|-\lambda \varsigma]}}{(\eta-\lambda)} \mathrm{d} \lambda, \\
& \bar{\tau}_{\zeta Z}(\varsigma, Y, s)=\frac{-\operatorname{sign}(Y)}{2 \pi \mathrm{i}} \int_{\Gamma_{\lambda}} \frac{C_{e} s \alpha_{-}^{*}(\eta) \lambda \mathrm{e}^{-s[\alpha(\lambda)|Y|-\lambda \varsigma]}}{(\eta-\lambda) \alpha_{-}^{*}(\lambda)} \mathrm{d} \lambda, \\
& \bar{W}(\varsigma, Y, s)=\frac{-\operatorname{sign}(Y)}{2 \pi \mathrm{i}} \int_{\Gamma_{\lambda}} \frac{\alpha_{-}^{*}(\eta) \mathrm{e}^{-s[\alpha(\lambda)|Y|-\lambda \varsigma]}}{(\eta-\lambda) \alpha_{-}^{*}(\lambda)} \mathrm{d} \lambda .
\end{aligned}
$$

The corresponding result of stress intensity factor expressed in the Laplace transform domain is

$$
\bar{K}(s)=-C_{e} \sqrt{2 s(1-b v)} \alpha_{-}^{*}(\eta) .
$$


If the propagating velocity of the crack $v=0$ which is correspondent to the stationary crack, then Eqs. (32)-(34) are reduced to

$$
\begin{aligned}
& \bar{\tau}_{Y Z}(X, Y, s)=\frac{1}{2 \pi \mathrm{i}} \int_{\Gamma_{\lambda}} \frac{C_{e} s \alpha_{-}(\eta) \alpha_{+}(\lambda) \mathrm{e}^{-s[\alpha(\lambda)|Y|-\lambda X]}}{(\eta-\lambda)} \mathrm{d} \lambda, \\
& \bar{\tau}_{X Z}(X, Y, s)=\frac{-\operatorname{sign}(Y)}{2 \pi \mathrm{i}} \int_{\Gamma_{\lambda}} \frac{C_{e} s \alpha_{-}(\eta) \lambda \mathrm{e}^{-s[\alpha(\lambda)|Y|-\lambda X]}}{(\eta-\lambda) \alpha_{-}(\lambda)} \mathrm{d} \lambda, \\
& \bar{W}(X, Y, s)=\frac{-\operatorname{sign}(Y)}{2 \pi \mathrm{i}} \int_{\Gamma_{\lambda}} \frac{\alpha_{-}(\eta) \mathrm{e}^{-s[\alpha(\lambda)|Y|-\lambda X]}}{(\eta-\lambda) \alpha_{-}(\lambda)} \mathrm{d} \lambda, \\
& \bar{K}(s)=-C_{e} \sqrt{2 s} \alpha_{-}(\eta) .
\end{aligned}
$$

\section{Relations in the transformed domain for different moving coordinate systems}

The superposition method can be applied successfully only if the fundamental solutions and the integral function of superposition are specified in the same coordinate system. The coordinate transformation relations of two different moving coordinate systems should be established to solve the interaction of diffracted waves generated from the other crack tip and the propagating crack. Consider two moving coordinate systems $(\xi, y)$ and $\left(\xi^{\prime}, y\right)$ whose constant extending velocities are $v_{A}$ and $v_{B}$, respectively. If a function specified in the $(\xi, y)$ coordinate system is represented in the Laplace transform domain as

$$
\bar{Q}(\xi, y, s)=s^{n} \mathrm{e}^{s a} \int F(\lambda) \mathrm{e}^{-s \alpha_{A}^{*}(\lambda) y+s \lambda \xi} \mathrm{d} \lambda,
$$

where $n$ is an arbitrary integer, and

$$
\alpha_{A}^{*}(\lambda)=\alpha_{A+}^{*}(\lambda) \alpha_{A-}^{*}(\lambda)=\sqrt{b+\lambda\left(1-b v_{A}\right)} \sqrt{b-\lambda\left(1+b v_{A}\right)} .
$$

Then this function can be transformed into the $\left(\xi^{\prime}, y\right)$ coordinate system with the following form

$$
\begin{aligned}
\bar{Q}\left(\xi^{\prime}, y, s\right)= & s^{n} \mathrm{e}^{s a} \int\left[1-\lambda\left(v_{A}-v_{B}\right)\right]^{n-1} F\left(\frac{-\lambda}{1-\lambda\left(v_{A}-v_{B}\right)}\right) \\
& \times \mathrm{e}^{-s \alpha_{B}^{*}(\lambda) y+s \lambda\left[\xi^{\prime}-a\left(v_{A}-v_{B}\right)\right]} \mathrm{d} \lambda,
\end{aligned}
$$

in which

$$
\alpha_{B}^{*}(\lambda)=\alpha_{B+}^{*}(\lambda) \alpha_{B-}^{*}(\lambda)=\sqrt{b+\lambda\left(1-b v_{B}\right)} \sqrt{b-\lambda\left(1+b v_{B}\right)} .
$$

\section{Transient solutions for a stationary finite crack}

The problem to be considered in this section is an infinite anisotropic medium containing a finite crack of length $l$ subjected to a horizontally polarized shear wave 
as shown in Fig. 1. The origins of two coordinate systems $(x, y)$ and $\left(x^{\prime}, y^{\prime}\right)$ are located at crack tips $A$ and $B$, respectively. At time $t=0$, an incident plane horizontally polarized shear wave arrives at the crack tip $A$. It is assumed that the stress intensity factors of two crack tips will not exceed the fracture toughness of the material. Hence, the finite crack does not propagate but remains stationary all time. The influence of finite length of the crack on the dynamic stress intensity factor will be analyzed and discussed in detail. The incident plane wave with an incident angle $\varphi$ is represented by the general form

$$
w^{\mathrm{i}}(x, y, t)=F\left(t+b_{0} x \cos \varphi-b_{0} y \sin \varphi\right),
$$

where

$$
b_{0}=\frac{1}{\cos \varphi} \sqrt{\frac{C_{44} \rho}{\left(C_{44} \tan \varphi-C_{45}\right)^{2}+C_{e}^{2}}}
$$

is the slowness of the plane wave in the specified direction of an anisotropic material, and

$$
F(t)=H(t) \int_{0}^{t} f(\tau) \mathrm{d} \tau
$$

The function $F$ is identically zero when its argument is negative, but is otherwise an arbitrary wave form. Thus, the medium ahead of the incident plane wave front is undisturbed. In Eq. (44), $H()$ denotes the Heaviside function and $\varphi(0 \leqslant \varphi \leqslant \pi / 2)$ is the angle of the negative $x$-axis and the normal to the wave front. From Eq. (2), the incident horizontally polarized shear wave expressed in Eq. (42) will give rise to the shear stress in the anisotropic medium as follows:

$$
\begin{aligned}
\tau_{y z}^{\mathrm{i}}(x, y, t)= & -b_{0}\left(C_{44} \sin \varphi-C_{45} \cos \varphi\right) f\left(t+b_{0} x \cos \varphi-b_{0} y \sin \varphi\right) \\
& \times H\left(t+b_{0} x \cos \varphi-b_{0} y \sin \varphi\right) .
\end{aligned}
$$

At time $t=0$, the incident plane wave front reaches the crack tip $A$ and is diffracted. Some time later, the incident plane wave will arrive at the crack tip $B$ and another diffracted wave will be induced. The diffracted waves will scatter back and forth between the crack tips $A$ and $B$ at a later time. The sequence of wave fronts of incident, reflected and diffracted waves in a short time period are shown in Fig. 2. In analyzing this problem, the multiple diffractions of stress waves by the finite crack must be taken into account. This is the most difficult part in analyzing the problem, and fundamental solutions provided in Section 2 will be very useful to overcome this difficulty. Since the idea of stress intensity factor is a well-established concept in fracture mechanics, and it represents the cornerstone of applied linear elastic fracture mechanics, we will focus our attentions mainly on the solution of the dynamic stress intensity factor in the following derivation.

Introduce a linear coordinate transformation as indicated in Eqs. (4)-(6) by setting $v=0$ for the stationary crack, then the configuration and coordinate systems as shown in Fig. 1 for anisotropic materials are changed to that presented in Fig. 3 for isotropic 

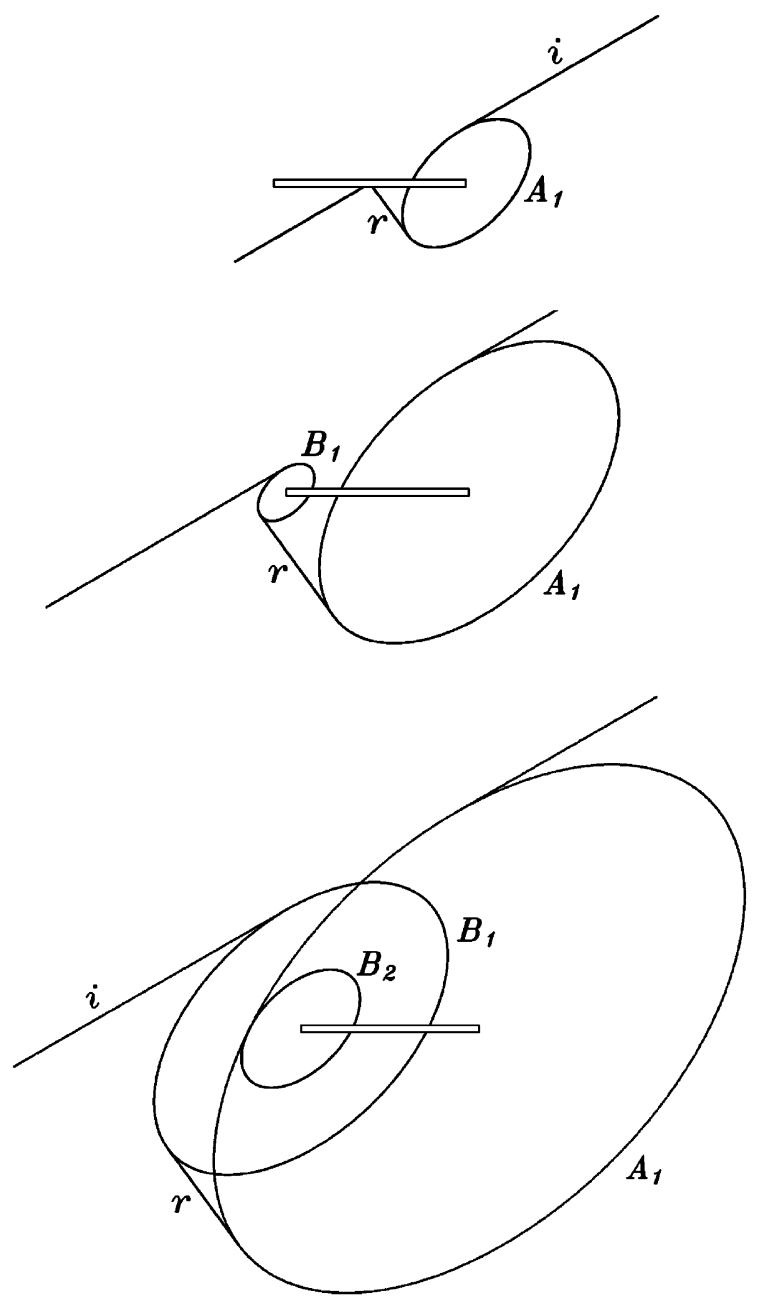

Fig. 2. Sequence of wave fronts of the scattered waves in a short time period for the stationary crack.

materials. It is interesting to see from Fig. 3 that the crack length remains $l$, but the incident angle and the velocity of plane wave are both changed. The new (transformed) incident angle and the slowness of the plane wave become

$$
\psi=\tan ^{-1}\left(\frac{C_{44} \tan \varphi}{C_{e}}-\frac{C_{45}}{C_{e}}\right)
$$

and

$$
b=\frac{b_{0} \cos \varphi}{C_{e}} \sqrt{\left(C_{44} \tan \varphi-C_{45}\right)^{2}+C_{e}^{2}},
$$




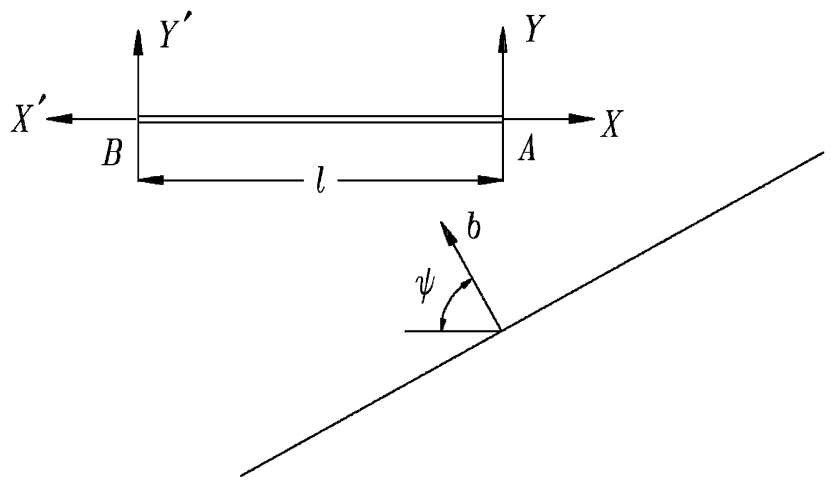

Fig. 3. Schematic and coordinate systems after using the linear coordinate transformation.

respectively. The incident shear stress in Eq. (45) can be rewritten as

$$
\tau_{Y Z}^{\mathrm{i}}(X, Y, t)=-b_{0} C_{\varphi} f(t+b X \cos \psi-b Y \sin \psi) H(t+b X \cos \psi-b Y \sin \psi),
$$

where

$$
C_{\varphi}=C_{44} \sin \varphi-C_{45} \cos \varphi .
$$

It is noted that

$$
\begin{aligned}
X^{\prime} & =x^{\prime}+\frac{C_{45}}{C_{44}} y^{\prime}, \\
Y^{\prime} & =\frac{C_{e}}{C_{44}} y^{\prime},
\end{aligned}
$$

and the relations of the field quantities in the $(X, Y)$ and $\left(X^{\prime}, Y^{\prime}\right)$ coordinates are

$$
\begin{aligned}
& \tau_{Y^{\prime} Z^{\prime}}\left(X^{\prime}, Y^{\prime}, t\right)=C_{e} \frac{\partial W^{\prime}\left(X^{\prime}, Y^{\prime}, t\right)}{\partial Y^{\prime}}, \\
& \tau_{X^{\prime} Z^{\prime}}\left(X^{\prime}, Y^{\prime}, t\right)=C_{e} \frac{\partial W^{\prime}\left(X^{\prime}, Y^{\prime}, t\right)}{\partial X^{\prime}}, \\
& W^{\prime}\left(X^{\prime}, Y^{\prime}, t\right)=-W(X, Y, t), \\
& \tau_{Y^{\prime} Z^{\prime}}\left(X^{\prime}, Y^{\prime}, t\right)=-\tau_{Y Z}(X, Y, t), \\
& \tau_{X^{\prime} Z^{\prime}}\left(X^{\prime}, Y^{\prime}, t\right)=\tau_{X Z}(X, Y, t) .
\end{aligned}
$$

The stress filed induced by the incident plane wave as indicated in Eq. (48) can be represented in the Laplace transform domain as

$$
\bar{\tau}_{Y Z}^{\mathrm{i}}(X, Y, s)=\frac{1}{2 \pi \mathrm{i}} \int_{\Gamma_{\lambda}} \frac{b_{0} C_{\varphi} \bar{f}(s)}{\lambda-b \cos \psi} \mathrm{e}^{-s \lambda Y \tan \psi+s \lambda X} \mathrm{~d} \lambda,
$$


or expressed in the $\left(X^{\prime}, Y^{\prime}\right)$ coordinate system as

$$
\bar{\tau}_{Y^{\prime} Z^{\prime}}^{\mathrm{i}}\left(X^{\prime}, Y^{\prime}, s\right)=\frac{1}{2 \pi \mathrm{i}} \int_{\Gamma_{\lambda}} \frac{b_{0} C_{\varphi} \bar{f}(s)}{\lambda+b \cos \psi} \mathrm{e}^{s \lambda Y^{\prime} \tan \psi+s \lambda\left(X^{\prime}+l\right)} \mathrm{d} \lambda .
$$

Here the overbar symbol is used for denoting the Laplace transform on time $t$. To ensure convergences of the complex integrals, we have the conditions that $\operatorname{Re}(\lambda-b \cos \psi)<0$ in Eq. (56) while $\operatorname{Re}(\lambda+b \cos \psi)>0$ in Eq. (57). Before the incident plane wave diffracted from the crack tip $B$, the stress field is precisely the same as that derived for a semi-infinite crack which lies in the plane $Y=0$ and $-\infty<X<0$, and is struck by the same incident plane wave. The incident stress field $\bar{\tau}_{Y Z}^{\mathrm{i}}(X, 0, s)$ at $Y=0$ generated by the horizontally polarized shear wave is

$$
\bar{\tau}_{Y Z}^{\mathrm{i}}(X, 0, s)=\frac{1}{2 \pi \mathrm{i}} \int_{\Gamma_{\lambda}} \frac{b_{0} C_{\varphi} \bar{f}(s)}{\lambda-b \cos \psi} \mathrm{e}^{s \lambda X} \mathrm{~d} \lambda .
$$

The applied traction on the crack face, in order to eliminate the incident wave as indicated in Eq. (58), has the functional form $\mathrm{e}^{s \lambda X}$. Since the solutions of applying traction $\mathrm{e}^{s \eta X}$ on crack faces in the transform domain have been solved in Section 2, the reflected and diffracted fields induced by the crack tip $A$ can be constructed by superimposing the incident wave traction that is equal and opposite to Eq. (58). When we combine Eqs. (58) and (27), the solution of displacement $\bar{w}^{A_{1}}$ for $A_{1}$ wave (the first wave diffracted from the crack tip $A$ ) can be expressed in the Laplace transform domain as follows:

$$
\begin{aligned}
\bar{W}^{A_{1}}(X, Y, s)= & \frac{-1}{2 \pi \mathrm{i}} \int_{\Gamma_{\eta_{1}}} \frac{b_{0} C_{\varphi} \bar{f}}{\eta_{1}-b \cos \psi} \\
& \times\left\{\frac{1}{2 \pi \mathrm{i}} \int_{\Gamma_{\eta_{2}}} \frac{-\operatorname{sign}(Y) \mathrm{e}^{-s \alpha\left(\eta_{2}\right)|Y|+s \eta_{2} X}}{C_{e} s \alpha_{+}\left(\eta_{1}\right)\left(\eta_{1}-\eta_{2}\right) \alpha_{-}\left(\eta_{2}\right)} \mathrm{d} \eta_{2}\right\} \mathrm{d} \eta_{1} \\
= & \frac{\operatorname{sign}(Y) b_{0} C_{\varphi} \bar{f}}{\sqrt{2 b} C_{e} \cos (\psi / 2) s} \frac{1}{2 \pi \mathrm{i}} \int_{\Gamma_{\lambda}} \frac{\mathrm{e}^{-s \alpha(\lambda)|Y|+s \lambda X}}{(\lambda-b \cos \psi) \alpha_{-}(\lambda)} \mathrm{d} \lambda .
\end{aligned}
$$

The corresponding stress intensity factor expressed in the Laplace transform domain is

$$
\bar{K}^{A_{1}}(s)=\frac{-1}{2 \pi \mathrm{i}} \int_{\Gamma_{\lambda}} \frac{b_{0} C_{\varphi} \bar{f}}{\lambda-b \cos \psi}\left\{\frac{-\sqrt{2}}{\sqrt{s} \alpha_{+}(\lambda)}\right\} \mathrm{d} \lambda=\frac{-b_{0} C_{\varphi} \bar{f}}{\sqrt{b} \cos (\psi / 2) \sqrt{s}} .
$$

By using the Cagniard-de Hoop method of Laplace inversion, the dynamic stress intensity factor of the crack tip $A$ induced by the incident wave will be

$$
K^{A_{1}}(t)=\frac{-b_{0} C_{\varphi}}{\sqrt{\pi b} \cos (\psi / 2)} \int_{0}^{t} \frac{f(\tau)}{\sqrt{t-\tau}} \mathrm{d} \tau .
$$


Subsequently, the incident plane wave will propagate toward to the crack tip $B$ and will be diffracted at time $t=b l \cos \psi$. Follow the similar procedure that is used for constructing the $A_{1}$ wave, the displacement induced by $B_{1}$ wave (the first wave diffracted from the crack tip $B)$ can be constructed in the coordinate system $\left(X^{\prime}, Y^{\prime}\right)$ by Eqs. (57) and (27) as follows

$$
\bar{W}^{B_{1}}\left(X^{\prime}, Y^{\prime}, s\right)=\frac{-\operatorname{sign}\left(Y^{\prime}\right) b_{0} C_{\varphi} \bar{f} \mathrm{e}^{-s b l \cos \psi}}{\sqrt{2 b} C_{e}|\sin (\psi / 2)| s} \frac{1}{2 \pi \mathrm{i}} \int_{\Gamma_{\lambda}} \frac{\mathrm{e}^{-s \alpha(\lambda)\left|Y^{\prime}\right|+s \lambda X^{\prime}}}{(\lambda+b \cos \psi) \alpha_{-}(\lambda)} \mathrm{d} \lambda .
$$

The corresponding stress intensity factor in the transform domain of crack tip $B$ is

$$
\bar{K}^{B_{1}}(s)=\frac{b_{0} C_{\varphi} \bar{f} \mathrm{e}^{-s b l \cos \psi}}{\sqrt{b}|\sin (\psi / 2)| \sqrt{s}} .
$$

The dynamic stress intensity factor at the crack tip $B$ expressed in time domain will be

$$
K^{B_{1}}(t)=\frac{b_{0} C_{\varphi}}{\sqrt{\pi b}|\sin (\psi / 2)|} \int_{0}^{t-b l \cos \psi} \frac{f(\tau)}{\sqrt{t-b l \cos \psi-\tau}} \mathrm{d} \tau .
$$

After the first incident plane wave arrives at crack tip $A$, the second wave that reaches the tip $A$ is the $B_{1}$ wave. When the diffracted $B_{1}$ wave arrives at the right tip $A$ of the finite crack at time $t=b l(1+\cos \psi)$, it carries a discontinuous displacement in the $Z$-direction which violates the displacement condition for $X>0$. In order to satisfy the condition that the displacement must be continuous for $X>0$, a distributed displacement is required to close the opening displacement. The diffracted $A_{2}$ wave will be induced when the $B_{1}$ wave arrives at the crack tip $A$ at time $t=b l(1+\cos \psi)$. To construct the $A_{2}$ wave, we change the formulation for the $B_{1}$ wave from $\left(X^{\prime}, Y^{\prime}\right)$ to $(X, Y)$ coordinate system, the displacement that we must eliminate ahead of the right $\operatorname{tip} A$ is

$$
\bar{W}^{B_{1}}(X, 0, s)=\frac{-b_{0} C_{\varphi} \bar{f} \mathrm{e}^{-s b l \cos \psi}}{\sqrt{2 b} C_{e}|\sin (\psi / 2)| s} \frac{1}{2 \pi \mathrm{i}} \int_{\Gamma_{\lambda}} \frac{\mathrm{e}^{-s \alpha(\lambda)|Y|+s \lambda(X+l)}}{(\lambda-b \cos \psi) \alpha_{+}(\lambda)} \mathrm{d} \lambda .
$$

The diffracted $A_{2}$ wave can be obtained by superimposing the distributed displacement that is equal and opposite to (65) in the Laplace transform domain as follows:

$$
\begin{aligned}
\bar{W}^{A_{2}}(X, Y, s)= & \frac{b_{0} C_{\varphi} \bar{f} \mathrm{e}^{-s b l \cos \psi}}{\sqrt{2 b} C_{e}|\sin (\psi / 2)| s} \frac{1}{2 \pi \mathrm{i}} \int_{\Gamma_{\eta_{1}}} \frac{\mathrm{e}^{s \eta_{1} l}}{\left(\eta_{1}-b \cos \psi\right) \alpha_{+}\left(\eta_{1}\right)} \\
& \times\left\{\frac{1}{2 \pi \mathrm{i}} \int_{\Gamma_{\eta_{2}}} \frac{-\operatorname{sign}(Y) \alpha_{-}\left(\eta_{1}\right) \mathrm{e}^{-s \alpha\left(\eta_{2}\right)|Y|+s \eta_{2} X}}{\left(\eta_{1}-\eta_{2}\right) \alpha_{-}\left(\eta_{2}\right)} \mathrm{d} \eta_{2}\right\} \mathrm{d} \eta_{1} \\
= & \frac{-\operatorname{sign}(Y) b_{0} C_{\varphi} \bar{f} \mathrm{e}^{-s b l \cos \psi}}{\sqrt{2 b} C_{e}|\sin (\psi / 2)| s} \frac{1}{(2 \pi \mathrm{i})^{2}} \\
& \times \int_{\Gamma_{\eta_{1}}} \int_{\Gamma_{\eta_{2}}} \frac{\alpha}{\left(\eta_{1}-b \cos \psi\right) \alpha_{+}\left(\eta_{1}\right)\left(\eta_{1}-\eta_{2}\right) \alpha_{-}\left(\eta_{2}\right)} \mathrm{d} \eta_{2} \mathrm{~d} \eta_{1} .
\end{aligned}
$$


Here Eq. (38) has been used to construct the solution of the diffracted $A_{2}$ wave. Using Eqs. (65) and (39), the corresponding stress intensity factor at crack tip $A$ due to the $A_{2}$ wave expressed in the Laplace transform domain will be

$$
\bar{K}^{A_{2}}(s)=\frac{-b_{0} C_{\varphi} \bar{f} \mathrm{e}^{-s b l \cos \psi}}{\sqrt{b}|\sin (\psi / 2)| \sqrt{s}} \frac{1}{2 \pi \mathrm{i}} \int_{\Gamma_{\lambda}} \frac{\alpha_{-}(\lambda) \mathrm{e}^{s \lambda l}}{(\lambda-b \cos \psi) \alpha_{+}(\lambda)} \mathrm{d} \lambda .
$$

The inversion Laplace transform of Eq. (67) will have the following form:

$$
\begin{aligned}
K^{A_{2}}(t)= & \frac{b_{0} C_{\varphi}}{\pi^{3 / 2} \sqrt{b}|\sin (\psi / 2)|} \int_{b l}^{t-b l \cos \psi} \int_{0}^{t-\tau-b l \cos \psi} \\
& \times \frac{f(\delta) \sqrt{\tau+b l}}{\sqrt{t-\tau-b l \cos \psi-\delta}(\tau+b l \cos \psi) \sqrt{\tau-b l}} \mathrm{~d} \delta \mathrm{d} \tau .
\end{aligned}
$$

By using the same process that is used for constructing $A_{2}$ wave, the diffracted $B_{2}$ wave, which is induced after the diffracted $A_{1}$ wave arrives at crack tip $B$ at time $t=b l$, can be obtained from Eqs. (59) and (38) and is expressed in the coordinate system of $\left(X^{\prime}, Y^{\prime}\right)$ as follows:

$$
\begin{aligned}
\bar{W}^{B_{2}}\left(X^{\prime}, Y^{\prime}, s\right)= & \frac{\operatorname{sign}\left(Y^{\prime}\right) b_{0} C_{\varphi} \bar{f}}{\sqrt{2 b} C_{e} \cos (\psi / 2) s} \frac{1}{(2 \pi \mathrm{i})^{2}} \\
& \times \int_{\Gamma_{\eta_{1}}} \int_{\Gamma_{\eta_{2}}} \frac{\alpha_{-}\left(\eta_{1}\right) \mathrm{e}^{s \eta_{1} l} \mathrm{e}^{-s \alpha\left(\eta_{2}\right)\left|Y^{\prime}\right|+s \eta_{2} X^{\prime}}}{\left(\eta_{1}+b \cos \psi\right) \alpha_{+}\left(\eta_{1}\right)\left(\eta_{1}-\eta_{2}\right) \alpha_{-}\left(\eta_{2}\right)} \mathrm{d} \eta_{2} \mathrm{~d} \eta_{1} .
\end{aligned}
$$

The correspondent stress intensity factor induced by the $B_{2}$ wave at the crack tip $B$ in the Laplace transform domain is

$$
\bar{K}^{B_{2}}(s)=\frac{b_{0} C_{\varphi} \bar{f}}{\sqrt{b} \cos (\psi / 2) \sqrt{s}} \frac{1}{2 \pi \mathrm{i}} \int_{\Gamma_{\lambda}} \frac{\alpha_{-}(\lambda) \mathrm{e}^{s \lambda l}}{(\lambda+b \cos \psi) \alpha_{+}(\lambda)} \mathrm{d} \lambda .
$$

Inversion of the Laplace transform yields

$$
K^{B_{2}}(t)=\frac{-b_{0} C_{\varphi}}{\pi^{3 / 2} \sqrt{b} \cos (\psi / 2)} \int_{b l}^{t} \int_{0}^{t-\tau} \frac{f(\delta) \sqrt{\tau+b l}}{\sqrt{t-\tau-\delta}(\tau-b l \cos \psi) \sqrt{\tau-b l}} \mathrm{~d} \delta \mathrm{d} \tau .
$$

The $B_{2}$ wave, which is generated by diffracting the $A_{1}$ wave at the left tip $B$ of the crack will return back to tip $A$ at time $t=2 b l$. After it arrives at tip $A$, the displacement boundary condition for $X>0$ will be violated. An appropriate displacement along $X>0$ must be superimposed to close the opening displacement ahead of the crack tip. Combine the results of Eqs. (69) and (39), the corresponding stress intensity factor for $\operatorname{tip} A$ in the Laplace transform domain can be obtained as follows:

$$
\bar{K}^{A_{3}}(s)=\frac{-b_{0} C_{\varphi} \bar{f}}{\sqrt{b} \cos (\psi / 2) \sqrt{s}} \frac{1}{(2 \pi \mathrm{i})^{2}} \int_{\Gamma_{\eta_{1}}} \int_{\Gamma_{\eta_{2}}} \frac{\alpha_{-}\left(\eta_{1}\right) G\left(\eta_{1}, \eta_{2}\right) \mathrm{e}^{s \eta_{1} l} \mathrm{e}^{s \eta_{2} l}}{\left(\eta_{1}+b \cos \psi\right) \alpha_{+}\left(\eta_{1}\right)} \mathrm{d} \eta_{2} \mathrm{~d} \eta_{1},
$$


where

$$
G\left(\eta_{1}, \eta_{2}\right)=\frac{\alpha_{-}\left(\eta_{2}\right)}{\left(\eta_{1}+\eta_{2}\right) \alpha_{-}\left(-\eta_{2}\right)}
$$

The inverse Laplace transform of Eq. (72) is

$$
\begin{aligned}
K^{A_{3}}(t)= & \frac{-b_{0} C_{\varphi}}{\pi^{5 / 2} \sqrt{b} \cos (\psi / 2)} \int_{2 b l}^{t} \int_{b l}^{\tau-b l} \int_{0}^{t-\tau} \frac{f(\delta)}{\sqrt{t-\tau-\delta}} \\
& \times\left[\frac{\sqrt{t_{1}+b l} \sqrt{t_{2}+b l}}{\left(t_{1}-b l \cos \psi\right) \sqrt{t_{1}-b l}\left(t_{1}+t_{2}\right) \sqrt{t_{2}-b l}}\right]_{t=\tau} \mathrm{d} \delta \mathrm{d} t_{1} \mathrm{~d} \tau,
\end{aligned}
$$

where

$$
t_{1}+t_{2}=t
$$

Similarly, the stress intensity factor at the crack tip $B$ due to the diffraction of $B_{3}$ wave at $t=b l(2+\cos \psi)$ is

$$
\begin{aligned}
K^{B_{3}}(t)= & \frac{b_{0} C_{\varphi}}{\pi^{5 / 2} \sqrt{b}|\sin (\psi / 2)|} \int_{2 b l}^{t-b l \cos \psi} \int_{b l}^{\tau-b l} \int_{0}^{t-\tau-b l \cos \psi} \frac{f(\delta)}{\sqrt{t-\tau-b l \cos \psi-\delta}} \\
& \times\left[\frac{\sqrt{t_{1}+b l} \sqrt{t_{2}+b l}}{\left(t_{1}+b l \cos \psi\right) \sqrt{t_{1}-b l}\left(t_{1}+t_{2}\right) \sqrt{t_{2}-b l}}\right]_{t=\tau} \mathrm{d} \delta \mathrm{d} t_{1} \mathrm{~d} \tau
\end{aligned}
$$

For the time being, we have constructed in detail for the first three diffracted waves which have contributions to the stress intensity factor at crack tips $A$ and $B$. The process of diffraction of the waves continues as time goes on. Following a similar procedure, the complete solutions for dynamic stress intensity factor at tips $A$ and $B$ that account for the contributions of the incident wave and all the diffracted waves induced from the two crack tips can be expressed explicitly as follows:

$$
\begin{aligned}
& K^{A}(t)=\sum_{n=1}^{\infty} K^{A_{n}}(t), \\
& K^{B}(t)=\sum_{n=1}^{\infty} K^{B_{n}}(t),
\end{aligned}
$$

where

$$
\begin{aligned}
K^{A_{1}}(t) & =\frac{-b_{0} C_{\varphi}}{\sqrt{\pi b} \cos (\psi / 2)} \int_{0}^{t} \frac{f(\tau)}{\sqrt{t-\tau}} \mathrm{d} \tau, \\
K^{B_{1}}(t) & =\frac{b_{0} C_{\varphi}}{\sqrt{\pi b}|\sin (\psi / 2)|} \int_{0}^{t-b l \cos \psi} \frac{f(\tau)}{\sqrt{t-b l \cos \psi-\tau}} \mathrm{d} \tau,
\end{aligned}
$$




$$
\begin{aligned}
K^{A_{2}}(t)= & \frac{b_{0} C_{\varphi}}{\pi^{3 / 2} \sqrt{b}|\sin (\psi / 2)|} \int_{b l}^{t-b l \cos \psi} \int_{0}^{t-\tau-b l \cos \psi} \\
& \times \frac{f(\delta) \sqrt{\tau+b l}}{\sqrt{t-\tau-b l \cos \psi-\delta}(\tau+b l \cos \psi) \sqrt{\tau-b l}} \mathrm{~d} \delta \mathrm{d} \tau, \\
K^{B_{2}}(t)= & \frac{-b_{0} C_{\varphi}}{\pi^{3 / 2} \sqrt{b} \cos (\psi / 2)} \int_{b l}^{t} \int_{0}^{t-\tau} \frac{f(\delta) \sqrt{\tau+b l}}{\sqrt{t-\tau-\delta(\tau-b l \cos \psi)} \sqrt{\tau-b l}} \mathrm{~d} \delta \mathrm{d} \tau, \\
K^{A_{n}}(t)= & \frac{(-1)^{n} b_{0} C_{\varphi}}{\sqrt{\pi} \pi^{n-1} \sqrt{b}[q|\sin (\psi / 2)|+(1-q) \cos (\psi / 2)]} \int_{(n-1) b l}^{t-q b l \cos \psi} \int_{b l}^{a_{1}} \int_{b l}^{a_{2}} \\
& \ldots \int_{b l}^{a_{n-2}} \int_{0}^{t-\tau-q b l \cos \psi} \frac{f(\delta)}{\sqrt{t-\tau-q b l \cos \psi-\delta}} \\
& \times \operatorname{SIFA~d~} \delta \mathrm{d} t_{n-2} \mathrm{~d} t_{n-3} \cdots \mathrm{d} t_{1} \mathrm{~d} \tau \quad \text { for } n=3,4,5, \ldots,
\end{aligned}
$$$$
\begin{aligned}
K^{B_{n}}(t)= & \frac{(-1)^{n-1} b_{0} C_{\varphi}}{\sqrt{\pi} \pi^{n-1} \sqrt{b}[(1-q)|\sin (\psi / 2)|+q \cos (\psi / 2)]} \\
& \times \int_{(n-1) b l \quad \int_{b l}^{t-(1-q) b l \cos \psi} \int_{b l}^{a_{1}} \cdots \int_{b l}^{a_{2}} \int_{0}^{a_{n-2}}}^{t-\tau-(1-q) b l \cos \psi} \\
& \times \frac{f(\delta)}{\sqrt{t-\tau-(1-q) b l \cos \psi-\delta}} \operatorname{SIFB} \mathrm{d} \delta \mathrm{d} t_{n-2} \mathrm{~d} t_{n-3} \cdots \mathrm{d} t_{1} \mathrm{~d} \tau
\end{aligned}
$$$$
\text { for } n=3,4,5, \ldots \text {, }
$$

in which

SIFA $=$ $\left[\frac{\sqrt{t_{1}+b l} \sqrt{t_{2}+b l} \cdots \sqrt{t_{n-1}+b l}}{\left[t_{1}+(-1)^{n} b l \cos \psi\right] \sqrt{t_{1}-b l}\left(t_{1}+t_{2}\right)\left(t_{2}+t_{3}\right) \cdots\left(t_{n-2}+t_{n-1}\right) \sqrt{t_{2}-b l} \sqrt{t_{3}-b l} \cdots \sqrt{t_{n-1}-b l}}\right]_{t=\tau}$, SIFB $=$

$$
\begin{aligned}
& {\left[\frac{\sqrt{t_{1}+b l} \sqrt{t_{2}+b l} \cdots \sqrt{t_{n-1}+b l}}{\left[t_{1}+(-1)^{n-1} b l \cos \psi\right] \sqrt{t_{1}-b l}\left(t_{1}+t_{2}\right)\left(t_{2}+t_{3}\right) \cdots\left(t_{n-2}+t_{n-1}\right) \sqrt{t_{2}-b l} \sqrt{t_{3}-b l} \cdots \sqrt{t_{n-1}-b l}}\right]_{t=\tau},} \\
& \quad a_{1}=\tau-(n-2) b l \\
& \quad a_{m}=\tau-t_{1}-t_{2}-\cdots-t_{m-1}-(n-m-1) b l \quad \text { for } m=2,3,4, \ldots, n-2, \\
& \quad q=0, \quad \text { when } n=3,5,7, \ldots ; \quad q=1, \quad \text { when } n=4,6,8, \ldots, \\
& t_{1}+t_{2}+t_{3}+\cdots+t_{n}=t .
\end{aligned}
$$


As a specific example, if we consider an incident step-stress wave for which

$$
f(t)=\frac{\tau_{0}}{C_{e} b_{0}}
$$

then the solutions can be simplified as

$$
\begin{aligned}
& K_{\mathrm{ss}}^{A}(t)=\sum_{n=1}^{\infty} K_{\mathrm{ss}}^{A_{n}}(t), \\
& K_{\mathrm{ss}}^{B}(t)=\sum_{n=1}^{\infty} K_{\mathrm{ss}}^{B_{n}}(t),
\end{aligned}
$$

where

$$
\begin{aligned}
& K_{\mathrm{ss}}^{A_{1}}(t)= \frac{-2 \tau_{0} C_{\varphi}}{C_{e} \cos (\psi / 2)} \sqrt{\frac{t}{\pi b}} H(t), \\
& K_{\mathrm{ss}}^{B_{1}}(t)= \frac{2 \tau_{0} C_{\varphi}}{C_{e}|\sin (\psi / 2)|} \sqrt{\frac{t-b l \cos \psi}{\pi b}} H(t-b l \cos \psi), \\
& K_{\mathrm{ss}}^{A_{2}}(t)= \frac{2 \tau_{0} C_{\varphi}}{\pi^{3 / 2} C_{e} \sqrt{b}|\sin (\psi / 2)|} \int_{b l}^{t-b l \cos \psi} \frac{\sqrt{t-\tau-b l \cos \psi} \sqrt{\tau+b l}}{(\tau+b l \cos \psi) \sqrt{\tau-b l}} \mathrm{~d} \tau, \\
& K_{\mathrm{ss}}^{B_{2}}(t)= \frac{-2 \tau_{0} C_{\varphi}}{\pi^{3 / 2} C_{e} \sqrt{b} \cos (\psi / 2)} \int_{b l}^{t} \frac{\sqrt{t-\tau} \sqrt{\tau+b l}}{(\tau-b l \cos \psi) \sqrt{\tau-b l}} \mathrm{~d} \tau, \\
& K_{\mathrm{ss}}^{A_{n}}(t)= \frac{(-1)^{n} 2 \tau_{0} C_{\varphi}}{\sqrt{\pi} \pi^{n-1} C_{e} \sqrt{b}[q|\sin (\psi / 2)|+(1-q) \cos (\psi / 2)]} \\
& \times \int_{(n-1) b l}^{t-q b l \cos \psi} \int_{b l}^{a_{1}} \int_{b l}^{a_{2}} \ldots \int_{b l}^{a_{n-2}} \sqrt{t-\tau-q b l \cos \psi} \\
& \times \operatorname{SIFA} \mathrm{d} t_{n-2} \mathrm{~d} t_{n-3} \cdots \mathrm{d} t_{1} \mathrm{~d} \tau \quad \text { for } n=3,4,5, \ldots, \\
& K_{\mathrm{ss}}^{B_{n}}(t)= \frac{(-1)^{n-1} 2 \tau_{0} C_{\varphi}}{\sqrt{\pi} \pi^{n-1} C_{e} \sqrt{b}[(1-q)|\sin (\psi / 2)|+q \cos (\psi / 2)]} \int_{(n-1) b l}^{t-(1-q) b l \cos \psi} \\
& \times \int_{b l}^{a_{1}} \int_{b l}^{a_{2}} \ldots \int_{b l}^{a_{n-2}} \sqrt{t-\tau-(1-q) b l \cos \psi} \\
& \operatorname{SIFB} \mathrm{d} t_{n-2} \mathrm{~d} t_{n-3} \cdots \mathrm{d} t_{1} \mathrm{~d} \tau \quad \text { for } n=3,4,5, \ldots \\
&
\end{aligned}
$$

The corresponding static solutions for the stress intensity factor due to a step-stress wave are

$$
K_{\mathrm{ss}}^{A, s}=-\frac{\tau_{0} C_{\varphi}}{C_{e}} \sqrt{\frac{\pi l}{2}}
$$




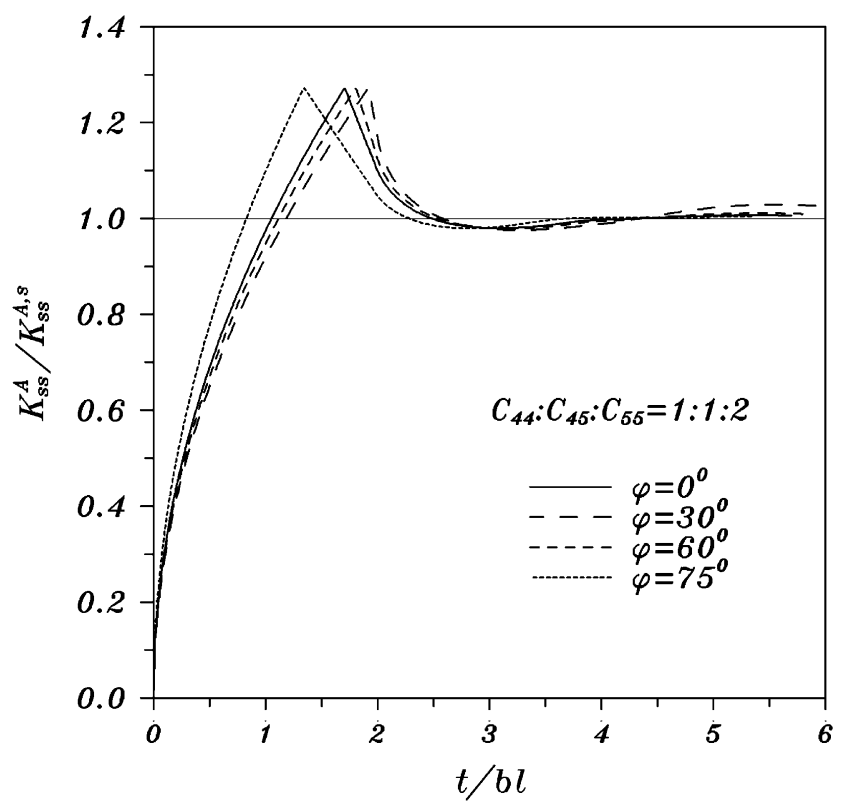

Fig. 4. Transient responses of the normalized stress intensity factors at crack tip $A$ for stationary cracks.

and

$$
K_{\mathrm{ss}}^{B, s}=\frac{\tau_{0} C_{\varphi}}{C_{e}} \sqrt{\frac{\pi l}{2}} .
$$

The exact transient solutions of dynamic stress intensity factors for multiple diffractions of a plane horizontally polarized shear wave by a stationary finite crack in an anisotropic medium have been derived. The induced wave fronts of incident, reflected and diffracted waves in a short time period are shown in Fig. 2. Numerical results for a step-stress wave case will be considered in the following discussion. Since the transient solution is exact up to the arrival time of the next wave, only a finite number of waves will be involved in the numerical calculation. The numerical calculation includes many high dimensional integrals and it is performed by using the 24-term Gaussian formula. Figs. 4 and 5 show the dimensionless dynamic stress intensity factors $K_{\mathrm{ss}}^{A} / K_{\mathrm{ss}}^{A, s}$ and $K_{\mathrm{ss}}^{B} / K_{\mathrm{ss}}^{B, s}$ at tips $A$ and $B$ versus the dimensionless time $t / b l$ for different values of the incident angle $\varphi$, respectively. It can be seen that the dynamic stress intensity factor will increase with time and will reach a maximum value just before the diffracted waves arrive at the crack tip. The stress intensity factors decrease after the diffracted waves induced by the other tip reach the crack tip, and then oscillate near the static value. The ratio of the value for maximum dynamic overshot can be calculated from Eqs. (80)-(83) as follows

$$
\frac{\left.K_{\mathrm{ss}}^{A_{1}}(t)\right|_{t=b l(1+\cos \psi)}}{K_{\mathrm{ss}}^{A, s}}=\frac{\left.K_{\mathrm{ss}}^{B_{1}}(t)\right|_{t=b l}}{K_{\mathrm{ss}}^{B, s}}=\frac{4}{\pi} .
$$




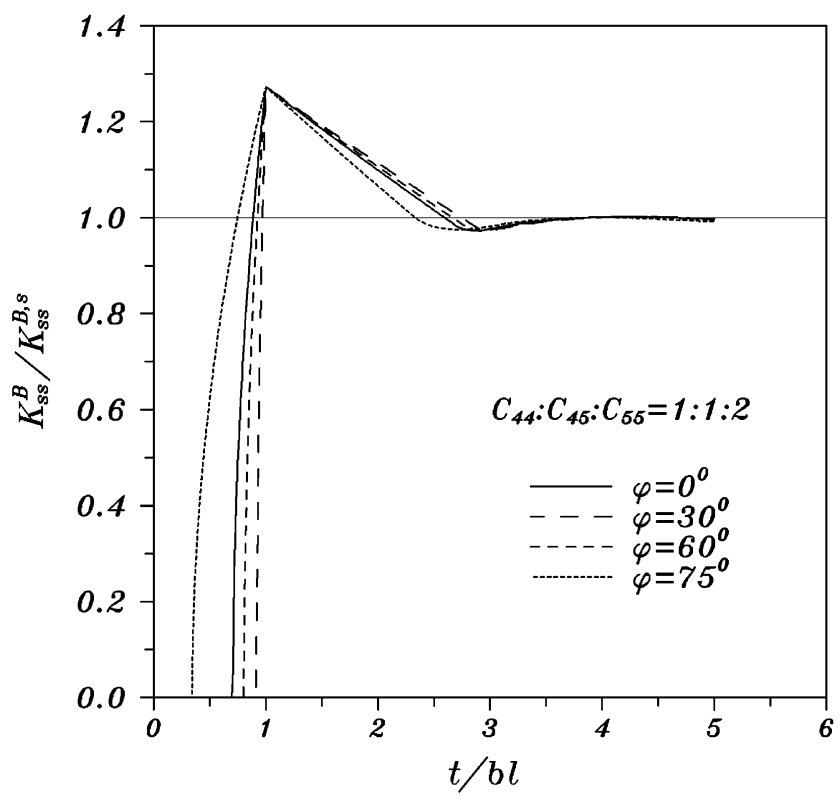

Fig. 5. Transient responses of the normalized stress intensity factors at crack tip $B$ for stationary cracks.

This result is the same as that for the isotropic case (Achenbach, 1970a). The maximum value of stress intensity factor at tips $A$ and $B$ is the same and has the following expression:

$$
\begin{aligned}
K_{\mathrm{ss}, \max }^{A} & =\left.K_{\mathrm{ss}}^{A_{1}}(t)\right|_{t=b l(1+\cos \psi)}=K_{\mathrm{ss}, \text { max }}^{B}=\left.K_{\mathrm{ss}}^{B_{1}}(t)\right|_{t=b l} \\
& =\frac{-2 \sqrt{2 l} \tau_{0} C_{\varphi}}{\sqrt{\pi} C_{e}} \\
& =\frac{2 \sqrt{2 l\left(C_{44}^{2}+C_{45}^{2}\right) \tau_{0}}}{\sqrt{\pi} C_{e}} \sin \left(\tan ^{-1}\left(\frac{C_{45}}{C_{44}}\right)-\varphi\right) .
\end{aligned}
$$

For small angle of $\varphi$ as indicated in Figs. 4 and 5, the stress intensity factor of crack tip $B$ increases more rapidly with time than that of crack tip $A$. The times that the maximum values of the overshot occur are $b l(1+\cos \psi)$ and $b l$ for crack tips $A$ and $B$, respectively. Because the time that the stress intensity factor of crack tip $B$ reaches its maximum value is shorter than that of tip $A$, it is very well possible that the crack tip $B$ will propagate earlier than crack tip $A$ for large fracture toughness of the material. Furthermore, if a pulse step-stress wave with a short duration time less than $b l(1+\cos \psi)$ is considered, then it is also possible that crack propagation will occur at crack tip $B$, but crack tip $A$ remains stationary.

Figs. 6 and 7 show the dimensionless stress intensity factors $K_{\mathrm{ss}}^{A} /\left(l^{1 / 2} \tau_{0}\right)$ and $K_{\mathrm{ss}}^{B} /\left(l^{1 / 2} \tau_{0}\right)$ versus the dimensionless time $t / b l$ for different values of the incident angle 


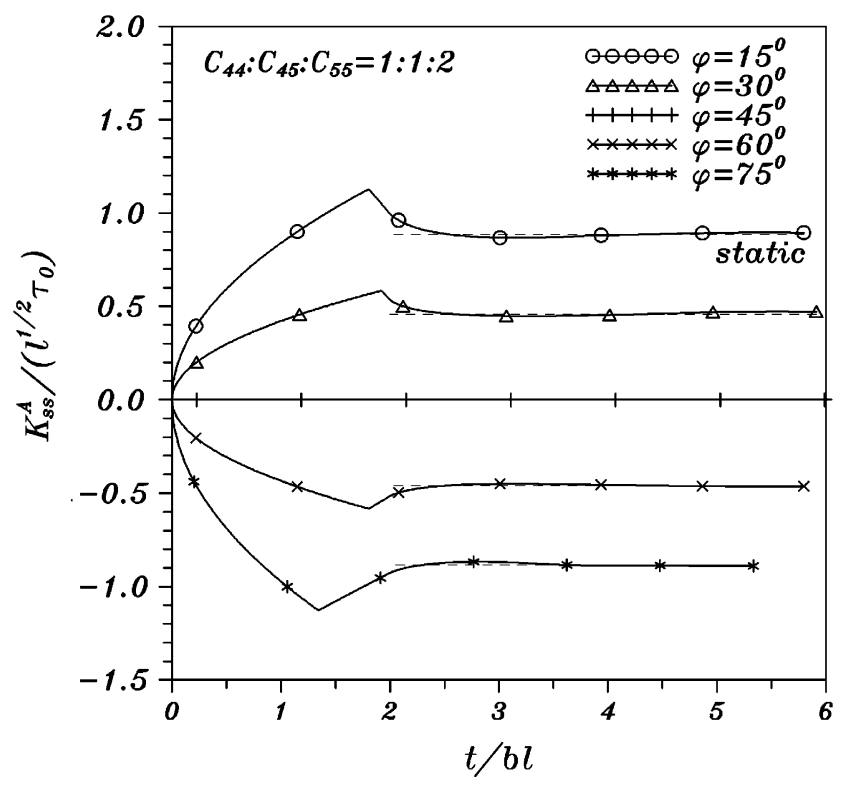

Fig. 6. Transient responses of the normalized stress intensity factors at crack tip $A$ for different incident angles.

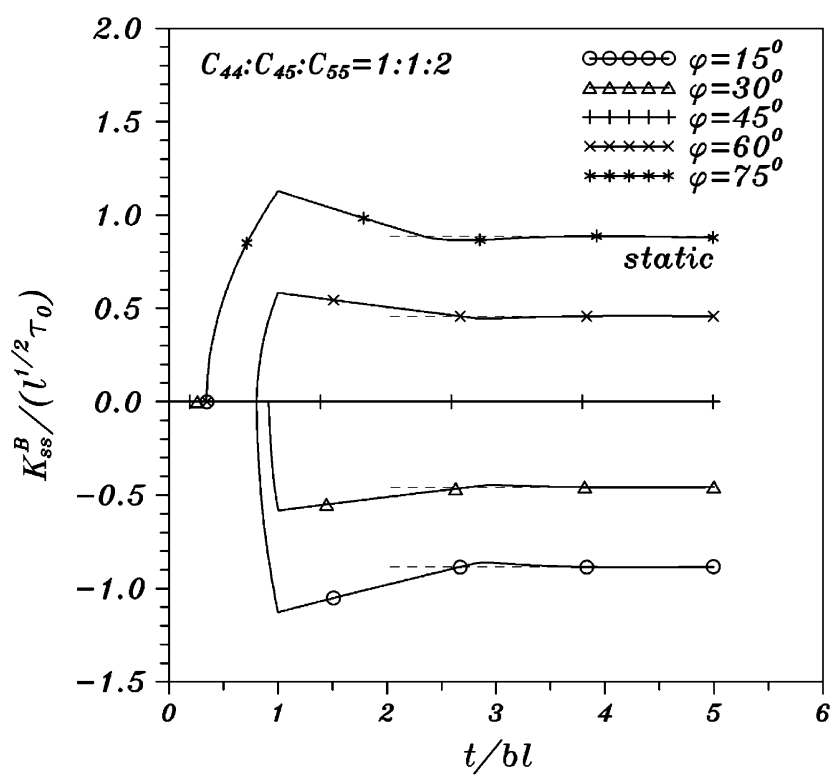

Fig. 7. Transient responses of the normalized stress intensity factors at crack tip $B$ for different incident angles. 


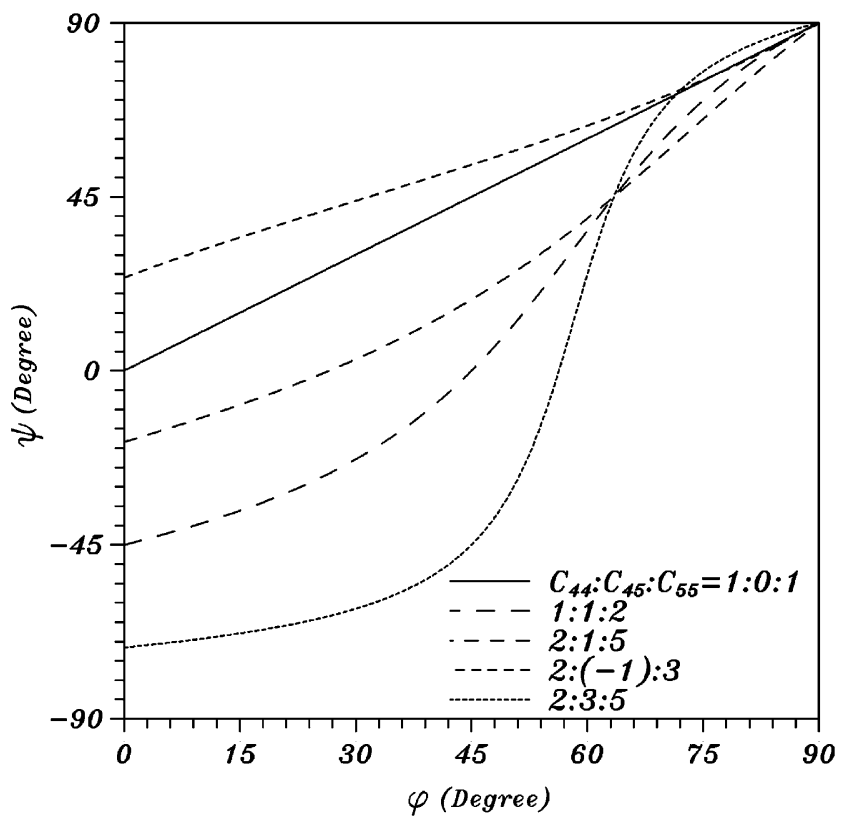

Fig. 8. The relations of $\psi$ and $\varphi$ for different material constants.

$\varphi$, respectively. It can be seen that the peak magnitude has no obvious relation with the incident angle $\varphi$. It is well known that the magnitude of the stress intensity factor increases as the incident angle $\varphi$ increases for an isotropic finite crack. However, it is not generally true for an anisotropic finite crack. It is indicated in Figs. 6 and 7 that the stress intensity factors of both tips are zero for $\varphi=45^{\circ}$. The reason is that the stress intensity factor is dependent on the transformed angle $\psi$ which is a function of $\varphi$ and material constants as indicated in Eq. (46). The transformed angle $\psi$ equals zero for $\varphi=45^{\circ}$ under the combination of material constants shown in Figs. 6 and 7. The functions $\psi(\varphi)$ in Eq. (46) for different material constants are plotted in Fig. 8 and the solid line represents the isotropic case. From Eq. (46), it is found that the transformed angle $\psi \geqslant 0$ when $\varphi \geqslant \tan ^{-1}\left(C_{45} / C_{44}\right)$, and $\psi<0$ when $\varphi<\tan ^{-1}\left(C_{45} / C_{44}\right)$. The special condition that will induce zero stress intensity factor for both tips is the incident angle $\varphi=\tan ^{-1}\left(C_{45} / C_{44}\right)$. For the isotropic material, we have $C_{45}=0$ and $\varphi=0$.

\section{Transient responses of a propagating finite crack}

In Section 4, the dynamic stress intensity factors of a stationary finite crack subjected to incident horizontally polarized shear waves in an unbounded anisotropic medium are investigated. It is assumed in Section 4 that the dynamic stress intensity factors of two crack tips are always smaller than the fracture toughness of the material, so 
that the propagation of the finite crack will not occur. In this section, however, the crack propagation event is considered and examined in detail. A similar geometry to be considered here is an infinite anisotropic medium containing a finite crack of length $l$. For time $t<0$, the finite crack is undisturbed. At time $t=0$, an incident horizontally polarized shear wave arrives at the crack tip $A$. The two crack tips are assumed to propagate along the crack tip line with different velocities at different time as the stress intensity factors reach the fracture toughness of the material. An incident step-stress wave (as given in Eq. (77)) is considered for the following analysis. The shear stress induced by the incident horizontally polarized shear wave can be represented in the $(X, Y)$ coordinate system as

$$
\tau_{Y Z}^{\mathrm{i}}(X, Y, t)=-\frac{\tau_{0} C_{\varphi}}{C_{e}} H(t+b X \cos \psi-b Y \sin \psi) .
$$

The stress filed in Eq. (86) can be expressed in the Laplace transform domain as

$$
\bar{\tau}_{Y Z}^{\mathrm{i}}(X, Y, s)=\frac{1}{2 \pi \mathrm{i}} \int_{\Gamma_{\lambda}} \frac{\tau_{0} C_{\varphi}}{C_{e} s(\lambda-b \cos \psi)} \mathrm{e}^{-s \lambda Y \tan \psi+s \lambda X} \mathrm{~d} \lambda,
$$

or expressed in the $\left(X^{\prime}, Y^{\prime}\right)$ coordinate system as

$$
\bar{\tau}_{Y^{\prime} Z^{\prime}}^{\mathrm{i}}\left(X^{\prime}, Y^{\prime}, s\right)=\frac{1}{2 \pi \mathrm{i}} \int_{\Gamma_{\lambda}} \frac{\tau_{0} C_{\varphi}}{C_{e} s(\lambda+b \cos \psi)} \mathrm{e}^{s \lambda Y^{\prime} \tan \psi+s \lambda\left(X^{\prime}+l\right)} \mathrm{d} \lambda .
$$

Apply the superposition method as indicated in Section 4, the displacement $\bar{W}^{A_{1 d}}$ for $A_{1 d}$ (the first wave diffracted from the stationary crack tip $A$ ) can be obtained from Eqs. (87) and (27), and the result is

$$
\bar{W}^{A_{1 d}}(X, Y, s)=\frac{\operatorname{sign}(Y) \tau_{0} C_{\varphi}}{\sqrt{2 b} C_{e}^{2} \cos (\psi / 2) s^{2}} \frac{1}{2 \pi \mathrm{i}} \int_{\Gamma_{\lambda}} \frac{\mathrm{e}^{-s \alpha(\lambda)|Y|+s \lambda X}}{(\lambda-b \cos \psi) \alpha_{-}(\lambda)} \mathrm{d} \lambda .
$$

The corresponding stress intensity factor is

$$
K^{A_{1 d}}(t)=\frac{-2 \tau_{0} C_{\varphi}}{C_{e} \cos (\psi / 2)} \sqrt{\frac{t}{\pi b}} H(t) .
$$

Eq. (90) is the well known solution of a stationary semi-infinite crack subjected to an incident plane wave. After some delay time $t_{\mathrm{f}}^{A}$, the dynamic stress intensity factor of tip $A$ reaches its fracture toughness $K_{\mathrm{c}}^{A}$ and the tip begins to propagate with a constant velocity along the crack tip line. The delay time $t_{\mathrm{f}}^{A}$ can be determined from Eq. (90) and yields

$$
t_{\mathrm{f}}^{A}=\pi b\left[\frac{C_{e} \cos (\psi / 2) K_{\mathrm{c}}^{A}}{2 \tau_{0} C_{\varphi}}\right]^{2} .
$$

Consequently, the fracture toughness $K_{\mathrm{c}}^{A}$ must be less than the maximum amplitude of dynamic stress intensity factor of tip $A$ during the transient process for stationary crack analysis, i.e., $K_{\mathrm{c}}^{A} \leqslant K_{\max }^{A}(t)$. It is known from the results for the previous section 
that the stress intensity factor of tip $A$ will reach its maximum amplitude at time $t=b l(1+\cos \psi)$. Hence, we have the condition for crack propagation that

$$
K_{\mathrm{c}}^{A} \leqslant K_{\max }^{A_{1 d}}(t)=\frac{2 \tau_{0}\left|C_{\varphi}\right|}{C_{e}} \sqrt{\frac{2 l}{\pi}}=K_{\mathrm{c}, \max }^{A} .
$$

At time $t=t_{\mathrm{f}}^{A}$, the dynamic stress intensity factor of crack tip $A$ reaches the fracture toughness and this tip starts to propagate with a constant subsonic velocity $v_{A}\left(b v_{A}<1\right)$. The incident field in Eq. (87) can be written in the Laplace transform domain for the moving coordinate system $(\varsigma, Y)$ as follows:

$$
\bar{\tau}_{Y Z}^{\mathrm{i}}(\varsigma, Y, s)=\frac{1}{2 \pi \mathrm{i}} \int_{\Gamma_{\lambda}} \frac{\tau_{0} C_{\varphi}\left(1+b v_{A} \cos \psi\right)}{C_{e} s\left[\left(1+b v_{A} \cos \psi\right) \lambda-b \cos \psi\right]} \mathrm{e}^{-s \lambda Y \tan \psi+s \lambda\left(\varsigma-v_{A} t_{\mathrm{f}}^{A}\right)} \mathrm{d} \lambda,
$$

where $\varsigma=X-v_{A}\left(t-t_{\mathrm{f}}^{A}\right)$. The applied traction on crack faces as indicated in Eq. (93) has the functional form $\mathrm{e}^{s \lambda \varsigma}$. The diffracted field generated from the propagating crack tip $A$ can be constructed by superimposing the fundamental solutions in Eqs. (21)(24) and the stress distribution in Eq. (93). The result of displacement expressed in the Laplace transform domain is

$$
\begin{aligned}
\bar{W}^{A_{1 v}}(\varsigma, Y, s)= & \frac{-1}{2 \pi \mathrm{i}} \int_{\Gamma_{\eta_{1}}} \frac{\tau_{0} C_{\varphi}\left(1+b v_{A} \cos \psi\right) \mathrm{e}^{-s \eta_{1} v_{A} t_{\mathrm{f}}^{A}}}{C_{e} s\left[\left(1+b v_{A} \cos \psi\right) \eta_{1}-b \cos \psi\right]} \\
& \times\left\{\frac{1}{2 \pi \mathrm{i}} \int_{\Gamma_{\eta_{2}}} \frac{-\operatorname{sign}(Y) \mathrm{e}^{-s \alpha_{A}^{*}\left(\eta_{2}\right)|Y|+s \eta_{2} \varsigma}}{C_{e} s \alpha_{A+}^{*}\left(\eta_{1}\right)\left(\eta_{1}-\eta_{2}\right) \alpha_{A-}^{*}\left(\eta_{2}\right)} \mathrm{d} \eta_{2}\right\} \mathrm{d} \eta_{1} \\
= & \frac{\operatorname{sign}(Y) \tau_{0} C_{\varphi}\left(1+b v_{A} \cos \psi\right)^{3 / 2} \mathrm{e}^{-s t_{A}}}{\sqrt{2 b} C_{e}^{2} \cos (\psi / 2) s^{2}} \\
& \times \frac{1}{2 \pi \mathrm{i}} \int_{\Gamma_{\lambda}} \frac{\mathrm{e}^{-s \alpha_{A}^{*}\left(\eta_{2}\right)|Y|+s \lambda \varsigma}}{\left[\left(1+b v_{A} \cos \psi\right) \lambda-b \cos \psi\right] \alpha_{A-}^{*}(\lambda)} \mathrm{d} \lambda,
\end{aligned}
$$

where

$$
t_{A}=\frac{b v_{A} t_{\mathrm{f}}^{A} \cos \psi}{1+b v_{A} \cos \psi} .
$$

The corresponding dynamic stress intensity factor for the propagating crack tip $A$ can also be constructed by a similar manner as follows:

$$
\begin{aligned}
\bar{K}^{A_{1 v}}(s) & =\frac{-1}{2 \pi \mathrm{i}} \int_{\Gamma_{\eta_{1}}} \frac{\tau_{0} C_{\varphi}\left(1+b v_{A} \cos \psi\right) \mathrm{e}^{-s v_{A} t_{\mathrm{f}}^{A}}}{C_{e} S\left[\left(1+b v_{A} \cos \psi\right) \lambda-b \cos \psi\right]}\left\{\frac{-\sqrt{2\left(1-b v_{A}\right)}}{\sqrt{s} \alpha_{A+}^{*}(\lambda)}\right\} \mathrm{d} \lambda \\
& =\frac{-\sqrt{\left(1-b v_{A}\right)\left(1+b v_{A} \cos \psi\right)} \tau_{0} C_{\varphi} \mathrm{e}^{-s t_{A}}}{\sqrt{b} C_{e} \cos (\psi / 2) s^{3 / 2}} .
\end{aligned}
$$


Apply the inverse Laplace transform to Eq. (95), the stress intensity factor for the propagating crack tip $A$ in time domain is

$$
\begin{aligned}
K^{A_{1 v}}(t) & =\frac{-2 \sqrt{\left(1-b v_{A}\right)\left(1+b v_{A} \cos \psi\right)} \tau_{0} C_{\varphi}}{\sqrt{\pi b} C_{e} \cos (\psi / 2)} \sqrt{t-t_{A}} H\left(t-t_{\mathrm{f}}^{A}\right) \\
& =\left(1-b v_{A}\right)^{1 / 2} \frac{-2 \tau_{0} C_{\varphi}}{C_{e} \cos (\psi / 2)} \sqrt{\frac{t+b v_{A} \cos \psi\left(t-t_{\mathrm{f}}^{A}\right)}{\pi b}} H\left(t-t_{\mathrm{f}}^{A}\right) \\
& =\left.\left(1-b v_{A}\right)^{1 / 2} K^{A_{1 d}}(t)\right|_{t=t+b v_{A} \cos \psi\left(t-t_{\mathrm{f}}^{A}\right)} H\left(t-t_{\mathrm{f}}^{A}\right) .
\end{aligned}
$$

The dynamic stress intensity factor expressed in Eq. (96) is the transient solution for a propagating semi-infinite crack subjected to the same incident step-stress wave. It has the interesting form of the product of a value $\left(1-b v_{A}\right)^{1 / 2}$ and the corresponding stress intensity factor $K^{A_{1 d}}(t)$ in Eq. (90) for a stationary crack by shifting the crack tip to the origin of the moving coordinate system $(\varsigma, Y)$. The value $\left(1-b v_{A}\right)^{1 / 2}$ is a universal function which depends only on the crack speed $v_{A}$ and material properties.

Subsequently, the incident plane wave will arrive at the crack tip $B$ at time $t=$ $b l \cos \psi$. Following the similar procedure that is used for constructing the $A_{1 d}$ wave, the $B_{1 d}$ wave (the first wave diffracted from the stationary crack tip $B$ ) can be constructed in the coordinate system $\left(X^{\prime}, Y^{\prime}\right)$ by using Eqs. (88) and (27) and yields

$$
\bar{W}^{B_{1 d}}\left(X^{\prime}, Y^{\prime}, s\right)=\frac{-\operatorname{sign}\left(Y^{\prime}\right) \tau_{0} C_{\varphi} \mathrm{e}^{-s b l \cos \psi}}{\sqrt{2 b} C_{e}^{2}|\sin (\psi / 2)| s^{2}} \frac{1}{2 \pi \mathrm{i}} \int_{\Gamma_{\lambda}} \frac{\mathrm{e}^{-s \alpha(\lambda)\left|Y^{\prime}\right|+s \lambda X^{\prime}}}{(\lambda+b \cos \psi) \alpha_{-}(\lambda)} \mathrm{d} \lambda .
$$

The corresponding dynamic stress intensity factor at the crack tip $B$ induced by the incident plane wave is

$$
K^{B_{1 d}}(t)=\frac{2 \tau_{0} C_{\varphi}}{C_{e}|\sin (\psi / 2)|} \sqrt{\frac{t-b l \cos \psi}{\pi b}} H(t-b l \cos \psi) .
$$

Similarly, when the dynamic stress intensity factor $K^{B_{1 d}}(t)$ reaches the fracture toughness $K_{\mathrm{c}}^{B}$ after some delay time $t_{\mathrm{f}}^{B}$, the crack tip $B$ will begin to propagate with a constant subsonic velocity $v_{B}\left(b v_{B}<1\right)$. The crack tip $B$ should start to propagate before the $A_{1 d}$ wave arrives the tip, i.e., $t_{\mathrm{f}}^{B}<b l$, as we have discussed in the previous section. The delay time $t_{\mathrm{f}}^{B}$ can be obtained from Eq. (98) and yields

$$
t_{\mathrm{f}}^{B}=\pi b\left(\frac{C_{e} \sin (\psi / 2) K_{\mathrm{c}}^{B}}{2 \tau_{0} C_{\varphi}}\right)^{2}+b l \cos \psi .
$$

The condition for the propagation of crack tip $B$ is that the fracture toughness $K_{\mathrm{c}}^{B}$ must be less than $K_{\max }^{B_{1 d}}(t)$, so we have

$$
K_{\mathrm{c}}^{B} \leqslant K_{\max }^{B_{1 d}}(t)=\frac{2 \tau_{0}\left|C_{\varphi}\right|}{C_{e}} \sqrt{\frac{2 l}{\pi}}=K_{\mathrm{c}, \max }^{B} .
$$


Similarly, the incident field expressed in Eq. (88) can be represented in the moving coordinate system $\left(\varsigma^{\prime}, Y^{\prime}\right)$ as

$$
\begin{aligned}
\bar{\tau}_{Y^{\prime} Z^{\prime}}^{\mathrm{i}}\left(\varsigma^{\prime}, Y^{\prime}, s\right)= & \frac{1}{2 \pi \mathrm{i}} \int_{\Gamma_{\lambda}} \frac{\tau_{0} C_{\varphi}\left(1-b v_{B} \cos \psi\right)}{C_{e} s\left[\left(1-b v_{B} \cos \psi\right) \lambda+b \cos \psi\right]} \\
& \times \mathrm{e}^{s \lambda Y^{\prime} \tan \psi+s \lambda\left(\varsigma^{\prime}-v_{B} t_{\mathrm{f}}^{B}+l\right)} \mathrm{d} \lambda,
\end{aligned}
$$

where $\varsigma^{\prime}=X^{\prime}-v_{B}\left(t-t_{\mathrm{f}}^{B}\right)$. The displacement field in the Laplace transform domain after the tip $B$ propagates can be obtained from Eqs. (101) and (23), and the result is

$$
\begin{aligned}
\bar{W}^{B_{1 v}}\left(\varsigma^{\prime}, Y^{\prime}, s\right)= & \frac{-\operatorname{sign}\left(Y^{\prime}\right) \tau_{0} C_{\varphi}\left(1-b v_{B} \cos \psi\right)^{3 / 2} \mathrm{e}^{-s t_{B}}}{\sqrt{2 b} C_{e}^{2}|\sin (\psi / 2)| s^{2}} \\
& \times \frac{1}{2 \pi \mathrm{i}} \int_{\Gamma_{\lambda}} \frac{\mathrm{e}^{-s \alpha_{B}^{*}\left(\eta_{2}\right)\left|Y^{\prime}\right|+s \lambda \varsigma^{\prime}}}{\left[\left(1-b v_{B} \cos \psi\right) \lambda+b \cos \psi\right] \alpha_{B-}^{*}(\lambda)} \mathrm{d} \lambda,
\end{aligned}
$$

where

$$
t_{B}=\frac{b \cos \psi\left(l-v_{B} t_{\mathrm{f}}^{B}\right)}{1-b v_{B} \cos \psi} .
$$

The stress intensity factor for the propagating crack tip $B$ in time domain can be obtained as follows:

$$
\begin{aligned}
K^{B_{1 v}}(t) & =\frac{2 \sqrt{\left(1-b v_{B}\right)\left(1-b v_{B} \cos \psi\right)} \tau_{0} C_{\varphi}}{\sqrt{\pi b} C_{e}|\sin (\psi / 2)|} \sqrt{t-t_{B}} H\left(t-t_{\mathrm{f}}^{B}\right) \\
& =\left(1-b v_{B}\right)^{1 / 2} \frac{2 \tau_{0} C_{\varphi}}{C_{e}|\sin (\psi / 2)|} \sqrt{\frac{t-b v_{B} \cos \psi\left(t-t_{\mathrm{f}}^{B}\right)-b l \cos \psi}{\pi b}} H\left(t-t_{\mathrm{f}}^{B}\right) \\
& =\left.\left(1-b v_{B}\right)^{1 / 2} K^{B_{1 d}}(t)\right|_{t=t-b v_{B} \cos \psi\left(t-t_{\mathrm{f}}^{B}\right)} H\left(t-t_{\mathrm{f}}^{B}\right) .
\end{aligned}
$$

The dynamic stress intensity factor expressed in Eq. (103) has the form of the product of a universal function $\left(1-b v_{B}\right)^{1 / 2}$ and the corresponding stress intensity factor $K^{B_{1 d}}(t)$ in Eq. (98) for a stationary crack by shifting the crack tip to the origin of the moving coordinate system $\left(\varsigma^{\prime}, Y^{\prime}\right)$.

At this point, the results and important features presented in this section are the same as that for the semi-infinite crack problem. The solutions are valid until the diffracted waves $\left(A_{1 d}\right.$ and $\left.B_{1 d}\right)$ induced from the other crack tip arrive at the propagating crack tips. The patterns of wave fronts for a short time after the crack tips propagate are shown in Fig. 9. To investigate the dynamic crack propagation event for the finite crack, the influence of the diffracted waves from the other crack tip on the propagating crack must be taken into account. Hence we will proceed the analysis a step further.

At a later time, the diffracted $B_{1 d}$ and $B_{1 v}$ waves generated from the crack tip $B$ will arrive at the propagating tip $A$ of the finite crack. The diffracted $A_{2 d}$ and $A_{2 v}$ waves will be induced as $B_{1 d}$ and $B_{1 v}$ waves arrive at the moving crack tip $A$, respectively. The displacement boundary condition for $\varsigma>0$ will not be satisfied and 

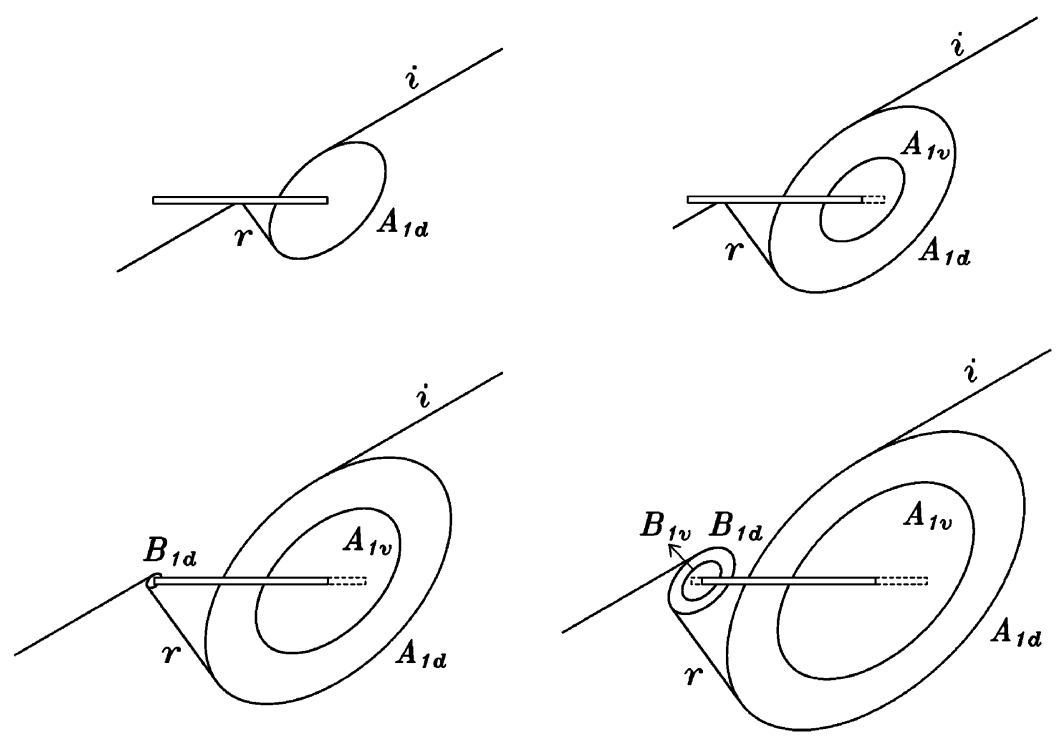

Fig. 9. Sequence of wave fronts of the scattered waves in a short time period for the propagating crack.

a distributed displacement is required to close the opening displacement ahead of the propagating crack tip. In order to superimpose with the fundamental solution, we change the formulations in Eqs. (97) and (102) to $(\varsigma, Y)$ coordinate system by using the transformation relations established in Section 3; the displacements we must eliminate ahead of the propagating tip $A$ are

$$
\begin{aligned}
\bar{W}^{B_{1 d}}(\varsigma, 0, s)= & \frac{-\tau_{0} C_{\varphi} \mathrm{e}^{-s b l \cos \psi}}{\sqrt{2 b} C_{e}^{2}|\sin (\psi / 2)| s^{2}} \\
& \times \frac{1}{2 \pi \mathrm{i}} \int_{\Gamma_{\lambda}} \frac{\mathrm{e}^{s \lambda\left(\varsigma-v_{A} t_{\mathrm{f}}^{A}+l+v_{A} b l \cos \psi\right)}}{\left(1-\lambda v_{A}\right)^{3 / 2}\left[\left(1+b v_{A} \cos \psi\right) \lambda-b \cos \psi\right] \alpha_{A+}^{*}(\lambda)} \mathrm{d} \lambda, \\
\bar{W}^{B_{1 v}}(\varsigma, 0, s)= & \frac{-\tau_{0} C_{\varphi}\left(1-b v_{B} \cos \psi\right)^{3 / 2} \mathrm{e}^{-s t_{B}}}{\sqrt{2 b} C_{e}^{2}|\sin (\psi / 2)| s^{2}} \\
& \times \frac{1}{2 \pi \mathrm{i}} \int_{\Gamma_{\lambda}} \frac{\mathrm{e}^{s \lambda\left(\varsigma-v_{A} t_{\mathrm{f}}^{A}-v_{B} t_{\mathrm{f}}^{B}+l+v_{\mathrm{r}} t_{B}\right)}}{\left(1-\lambda v_{\mathrm{r}}\right)^{3 / 2}\left[\left(1+b v_{A} \cos \psi\right) \lambda-b \cos \psi\right] \alpha_{A+}^{*}(\lambda)} \mathrm{d} \lambda,
\end{aligned}
$$

where $v_{\mathrm{r}}=v_{A}+v_{B}$ is the relative velocity between the two moving coordinate systems. The diffracted $A_{2 d}$ and $A_{2 v}$ waves can be obtained by superimposing the distributed displacement that is equal and opposite to Eqs. (104) and (105) ahead of the moving 
crack tip $\varsigma>0$ in the Laplace transform domain, respectively. By using Eqs. (104), (105) and (35), the corresponding stress intensity factors can be obtained as follows:

$$
\begin{aligned}
\bar{K}^{A_{2 d}}(s)= & \frac{-\sqrt{1-b v_{A}} \tau_{0} C_{\varphi} \mathrm{e}^{-s b l \cos \psi}}{\sqrt{b} C_{e}|\sin (\psi / 2)| s^{3 / 2}} \\
& \times \frac{1}{2 \pi \mathrm{i}} \int_{\Gamma_{\lambda}} \frac{\alpha_{A-}^{*}(\lambda) \mathrm{e}^{s \lambda\left(l-v_{A} t_{\mathrm{f}}^{A}+v_{A} b l \cos \psi\right)}}{\left(1-\lambda v_{A}\right)^{3 / 2}\left[\left(1+b v_{A} \cos \psi\right) \lambda-b \cos \psi\right] \alpha_{A+}^{*}(\lambda)} \mathrm{d} \lambda, \\
\bar{K}^{A_{2 v}}(s)= & \frac{-\sqrt{1-b v_{A}}\left(1-b v_{B} \cos \psi\right)^{3 / 2} \tau_{0} C_{\varphi} \mathrm{e}^{-s t_{B}}}{\sqrt{b} C_{e}|\sin (\psi / 2)| s^{3 / 2}} \\
& \times \frac{1}{2 \pi \mathrm{i}} \int_{\Gamma_{\lambda}} \frac{\left.\alpha_{A-}^{*}(\lambda) \mathrm{e}^{s \lambda\left(l-v_{A} t_{\mathrm{f}}^{A}-v_{B} t_{\mathrm{f}}^{B}+v_{\mathrm{r}} t_{B}\right.}\right)}{\left(1-\lambda v_{\mathrm{r}}\right)^{3 / 2}\left[\left(1+b v_{A} \cos \psi\right) \lambda-b \cos \psi\right] \alpha_{A+}^{*}(\lambda)} \mathrm{d} \lambda,
\end{aligned}
$$

Apply the inverse Laplace transform to Eqs. (106) and (107), the stress intensity factors in time domain will be

$$
\begin{aligned}
& K^{A_{2 d}}(t)=\frac{2 \sqrt{1-b v_{A}} \tau_{0} C_{\varphi}}{\pi^{3 / 2} \sqrt{b} C_{e}|\sin (\psi / 2)|} \int_{\frac{b\left(l-v_{A} t_{\mathrm{f}}^{A}+v_{A} b l \cos \psi\right)}{1-b v_{A}}}^{t-b l \cos \psi} \frac{\sqrt{t-\tau-b l \cos \psi}}{\left[v_{A} \tau+l-v_{A} t_{\mathrm{f}}^{A}+v_{A} b l \cos \psi\right]^{3 / 2}} \\
& \times \frac{\sqrt{\left(1+b v_{A}\right) \tau+b\left(l-v_{A} t_{\mathrm{f}}^{A}+v_{A} b l \cos \psi\right)}}{\left[\left(1+b v_{A} \cos \psi\right) \tau+b \cos \psi\left(l-v_{A} t_{\mathrm{f}}^{A}+v_{A} b l \cos \psi\right)\right]} \\
& \times \frac{\left(l-v_{A} t_{\mathrm{f}}^{A}+v_{A} b l \cos \psi\right)^{3 / 2}}{\sqrt{\left(1-b v_{A}\right) \tau-b\left(l-v_{A} t_{\mathrm{f}}^{A}+v_{A} b l \cos \psi\right)}} \mathrm{d} \tau H\left(t-t_{A_{2 d}}\right), \\
& K^{A_{2 v}}(t)=\frac{2 \sqrt{1-b v_{A}}\left(1-b v_{B} \cos \psi\right)^{3 / 2} \tau_{0} C_{\varphi}}{\pi^{3 / 2} \sqrt{b} C_{e}|\sin (\psi / 2)|} \\
& \times \int_{\frac{b\left(l-v_{A} t_{\mathrm{f}}^{A}-v_{B} t_{\mathrm{f}}^{B}+v_{\mathrm{r}} t_{B}\right)}{1-b v_{A}}}^{t-t_{B}} \frac{\sqrt{t-\tau-t_{B}}}{\left(v_{\mathrm{r}} \tau+l-v_{A} t_{\mathrm{f}}^{A}-v_{B} t_{\mathrm{f}}^{B}+v_{\mathrm{r}} t_{B}\right)^{3 / 2}} \\
& \times \frac{\sqrt{\left(1+b v_{A}\right) \tau+b\left(l-v_{A} t_{\mathrm{f}}^{A}-v_{B} t_{\mathrm{f}}^{B}+v_{\mathrm{r}} t_{B}\right)}}{\left[\left(1+b v_{A} \cos \psi\right) \tau+b \cos \psi\left(l-v_{A} t_{\mathrm{f}}^{A}-v_{B} t_{\mathrm{f}}^{B}+v_{\mathrm{r}} t_{B}\right)\right]} \\
& \times \frac{\left(l-v_{A} t_{\mathrm{f}}^{A}-v_{B} t_{\mathrm{f}}^{B}+v_{\mathrm{r}} t_{B}\right)^{3 / 2}}{\sqrt{\left(1-b v_{A}\right) \tau-b\left(l-v_{A} t_{\mathrm{f}}^{A}-v_{B} t_{\mathrm{f}}^{B}+v_{\mathrm{r}} t_{B}\right)}} \mathrm{d} \tau H\left(t-t_{A_{2 v}}\right),
\end{aligned}
$$

where

$$
t_{A_{2 d}}=\frac{b l \cos \psi+b\left(l-v_{A} t_{\mathrm{f}}^{A}\right)}{1-b v_{A}}
$$


and

$$
t_{A_{2 v}}=\frac{b\left(l-v_{A} t_{\mathrm{f}}^{A}\right)+t_{\mathrm{f}}^{B}}{1-b v_{A}}
$$

are the arrival time of the $B_{1 d}$ and $B_{1 v}$ waves at the propagating crack tip $A$, respectively.

Similarly, the $B_{2 d}$ and the $B_{2 v}$ diffracted waves scattering from the propagating crack tip $B$ will be induced after the $A_{1 d}$ and the $A_{1 v}$ waves arrive the propagating tip $B$, respectively. The dynamic stress intensity factors for the $B_{2 d}$ and the $B_{2 v}$ waves can be obtained as follows:

$$
\begin{aligned}
\bar{K}^{B_{2 d}}(s)= & \frac{\sqrt{1-b v_{B}} \tau_{0} C_{\varphi}}{\sqrt{b} C_{e} \cos (\psi / 2) s^{3 / 2}} \\
& \times \frac{1}{2 \pi \mathrm{i}} \int_{\Gamma_{\lambda}} \frac{\alpha_{B-}^{*}(\lambda) \mathrm{e}^{s \lambda\left(l-V_{B} t_{\mathrm{f}}^{B}\right)}}{\left(1-\lambda v_{B}\right)^{3 / 2}\left[\left(1-b v_{B} \cos \psi\right) \lambda+b \cos \psi\right] \alpha_{B+}^{*}(\lambda)} \mathrm{d} \lambda, \\
\bar{K}^{B_{2 v}}(s)= & \frac{\sqrt{1-b v_{B}}\left(1+b v_{A} \cos \psi\right)^{3 / 2} \tau_{0} C_{\varphi} \mathrm{e}^{-s t_{A}}}{\sqrt{b} C_{e} \cos (\psi / 2) s^{3 / 2}} \\
& \times \frac{1}{2 \pi \mathrm{i}} \int_{\Gamma_{\lambda}} \frac{\alpha_{B-}^{*}(\lambda) \mathrm{e}^{s \lambda\left(l-v_{A} t_{\mathrm{f}}^{A}-v_{B} t_{\mathrm{f}}^{B}+v_{\mathrm{r}} t_{A}\right)}}{\left(1-\lambda v_{\mathrm{r}}\right)^{3 / 2}\left[\left(1-b v_{B} \cos \psi\right) \lambda+b \cos \psi\right] \alpha_{B+}^{*}(\lambda)} \mathrm{d} \lambda .
\end{aligned}
$$

The inverse Laplace transform of Eqs. (112) and (113) are

$$
\begin{aligned}
K^{B_{2 d}}(t)= & \frac{-2 \sqrt{1-b v_{B}} \tau_{0} C_{\varphi}}{\pi^{3 / 2} \sqrt{b} C_{e} \cos (\psi / 2)} \\
& \times \int_{t_{B_{2 d}}}^{t} \frac{\sqrt{t-\tau} \sqrt{\left(1+b v_{B}\right) \tau+b\left(l-v_{B} t_{\mathrm{f}}^{B}\right)}}{\left(v_{B} \tau+l-v_{B} t_{\mathrm{f}}^{B}\right)^{3 / 2}\left[\left(1-b v_{B} \cos \psi\right) \tau-b \cos \psi\left(l-v_{B} t_{\mathrm{f}}^{B}\right)\right]} \\
& \times \frac{\left(l-v_{B} t_{\mathrm{f}}^{B}\right)^{3 / 2}}{\sqrt{\left(1-b v_{B}\right) \tau-b\left(l-v_{B} t_{\mathrm{f}}^{B}\right)}} \mathrm{d} \tau H\left(t-t_{B_{2 d}}\right), \\
K^{B_{2 v}}(t)= & \frac{-2 \sqrt{1-b v_{B}}\left(1+b v_{A} \cos \psi\right)^{3 / 2} \tau_{0} C_{\varphi}}{\pi^{3 / 2} \sqrt{b} C_{e} \cos (\psi / 2)} \\
& \times \int_{\frac{b\left(l-v_{A} t_{\mathrm{f}}^{A}-v_{B} t_{\mathrm{f}}^{B}+v_{\mathrm{r}} t_{A}\right)}{1-b v_{B}}}^{t-t_{A}} \frac{\sqrt{t-\tau-t_{A}}}{\left(v_{\mathrm{r}} \tau+l-v_{A} t_{\mathrm{f}}^{A}-v_{B} t_{\mathrm{f}}^{B}+v_{\mathrm{r}} t_{A}\right)^{3 / 2}}
\end{aligned}
$$




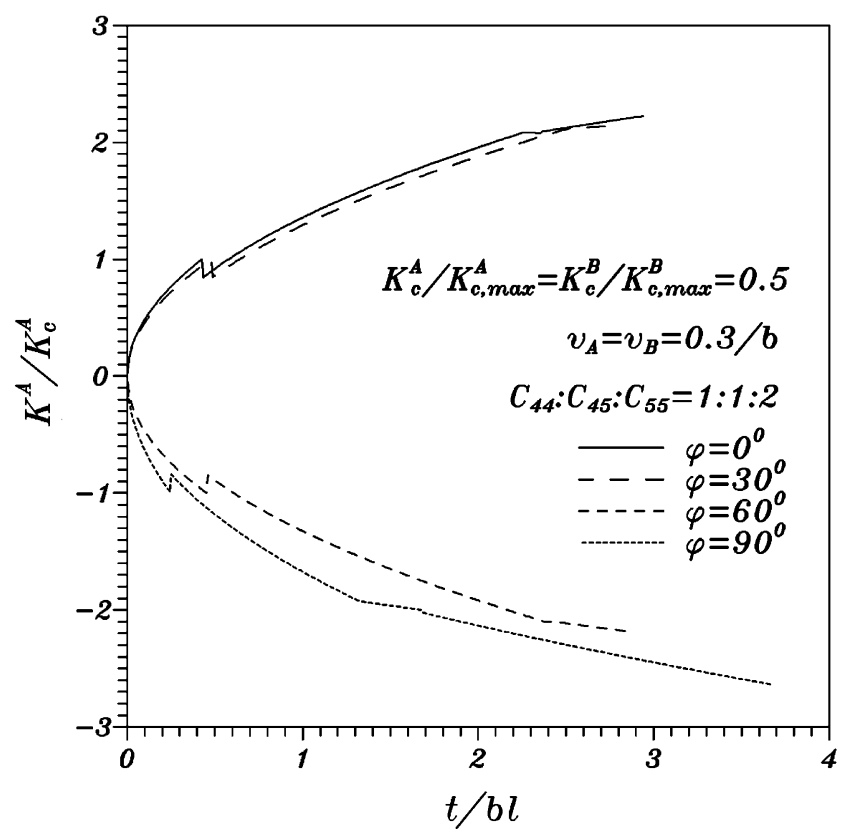

Fig. 10. Transient responses of the normalized stress intensity factors at propagating crack tip $A$ for different incident angles.

$$
\begin{aligned}
& \times \frac{\sqrt{\left(1+b v_{B}\right) \tau+b\left(l-v_{A} t_{\mathrm{f}}^{A}-v_{B} t_{\mathrm{f}}^{B}+v_{\mathrm{r}} t_{A}\right)}}{\left[\left(1-b v_{B} \cos \psi\right) \tau-b \cos \psi\left(l-v_{A} t_{\mathrm{f}}^{A}-v_{B} t_{\mathrm{f}}^{B}+v_{\mathrm{r}} t_{A}\right)\right]} \\
& \times \frac{\left(l-v_{A} t_{\mathrm{f}}^{A}-v_{B} t_{\mathrm{f}}^{B}+v_{\mathrm{r}} t_{A}\right)^{3 / 2}}{\sqrt{\left(1-b v_{B}\right) \tau-b\left(l-v_{A} t_{\mathrm{f}}^{A}-v_{B} t_{\mathrm{f}}^{B}+v_{\mathrm{r}} t_{A}\right)}} \mathrm{d} \tau H\left(t-t_{B_{2 v}}\right),
\end{aligned}
$$

where

$$
t_{B_{2 d}}=\frac{b\left(l-v_{B} t_{\mathrm{f}}^{B}\right)}{1-b v_{B}}
$$

and

$$
t_{B_{2 v}}=\frac{b\left(l-v_{B} t_{\mathrm{f}}^{B}\right)+t_{\mathrm{f}}^{A}}{1-b v_{B}}
$$

are the arrival times of the $A_{1 d}$ and $A_{1 v}$ waves at the propagating crack tip $B$, respectively.

Numerical calculation will be carried out and discussed according to the analytical solutions obtained in this section. Figs. 10 and 11 show the dimensionless stress intensity factors $K^{A} / K_{\mathrm{c}}^{A}$ and $K^{B} / K_{\mathrm{c}}^{B}$ versus the dimensionless time $t / b l$ for different values of the incident angle $\varphi$ for crack tip $A$ and $B$, respectively. Figs. 12 and 13 plot the dimensionless stress intensity factors $K^{A} / K_{\mathrm{c}}^{A}$ and $K^{B} / K_{\mathrm{c}}^{B}$ versus the dimensionless time 


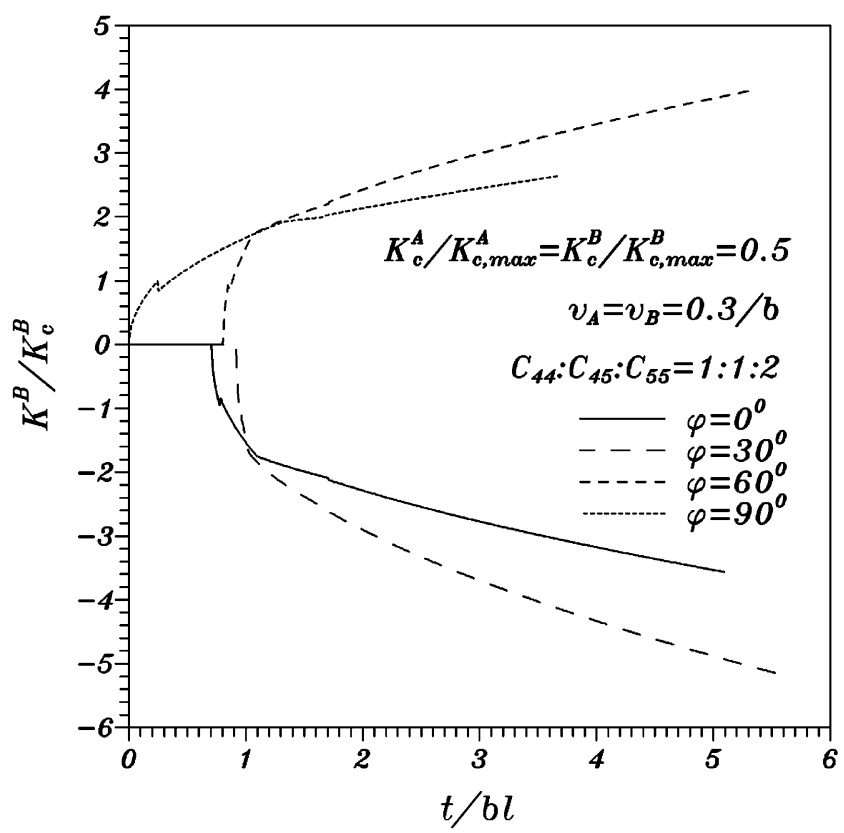

Fig. 11. Transient responses of the normalized stress intensity factors at propagating crack tip $B$ for different incident angles.

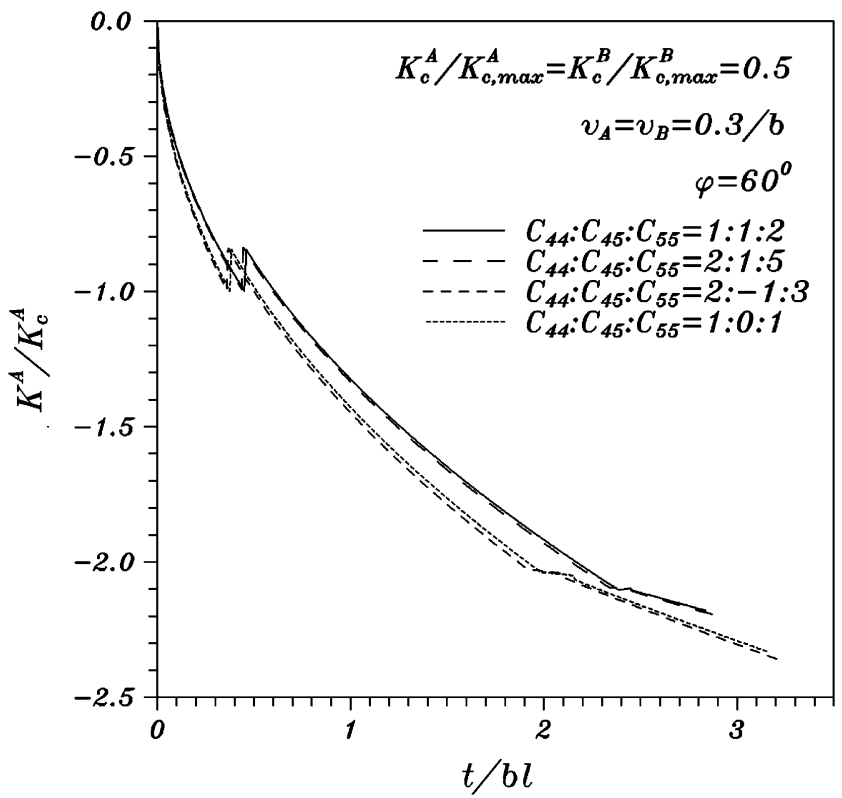

Fig. 12. Transient responses of the normalized stress intensity factors at propagating crack tip $A$ for different material constants. 


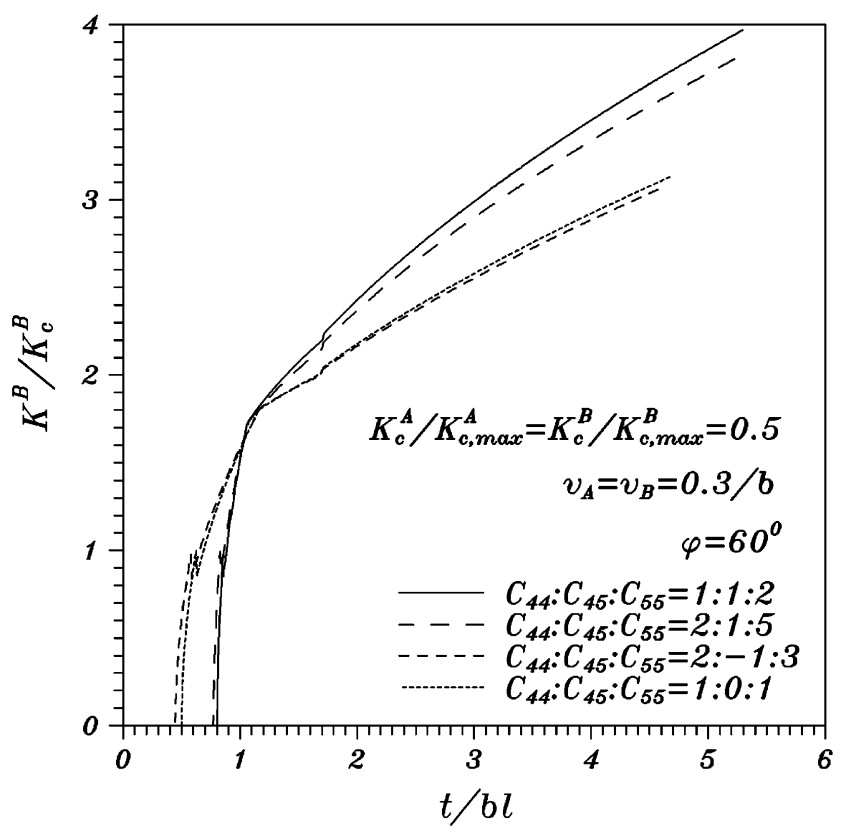

Fig. 13. Transient responses of the normalized stress intensity factors at propagating crack tip $B$ for different material constants.

$t / b l$ for different combinations of material constants, respectively. It is seen from these figures that the magnitudes of stress intensity factors increase before the crack begins to propagate, and arise a finite jump at the moment that the crack propagates and then continue the same tendency as the stationary crack. As indicated in Fig. 11 and Fig. 13, the two diffracted waves $\left(A_{1 d}\right.$ and $\left.A_{1 v}\right)$ generated from crack tip $A$ have very significant influence on the stress intensity factor for the propagating crack tip $B$. Since the stress intensity factor for crack tip $B$ increases more rapidly than the crack tip $A$ before the crack propagation, which results the dynamic stress intensity factor for crack tip $B$ is greater than the crack tip $A$ after the crack propagation. In order to get better understanding of whether the stress intensity factor at tip $B$ is larger than that at tip $A$, the values of the ratios of $K^{A_{1 v}}(t) / K^{B_{1 v}}(t)$ at the time that the first diffracted wave $A_{1 d}$ generated from the crack tip $A$ arrives at the propagating crack tip $B$ for different transformed angle $\psi$ are plotted in Figs. 14 and 15. It can be seen from these two figures that the value of $K^{B_{1 v}}$ is always larger than that of $K^{A_{1 v}}$ for any combination of fracture toughness and crack speeds.

\section{Conclusions}

The transient responses of a finite crack in an unbounded anisotropic medium subjected to an incident horizontally polarized shear wave are analyzed for stationary and 


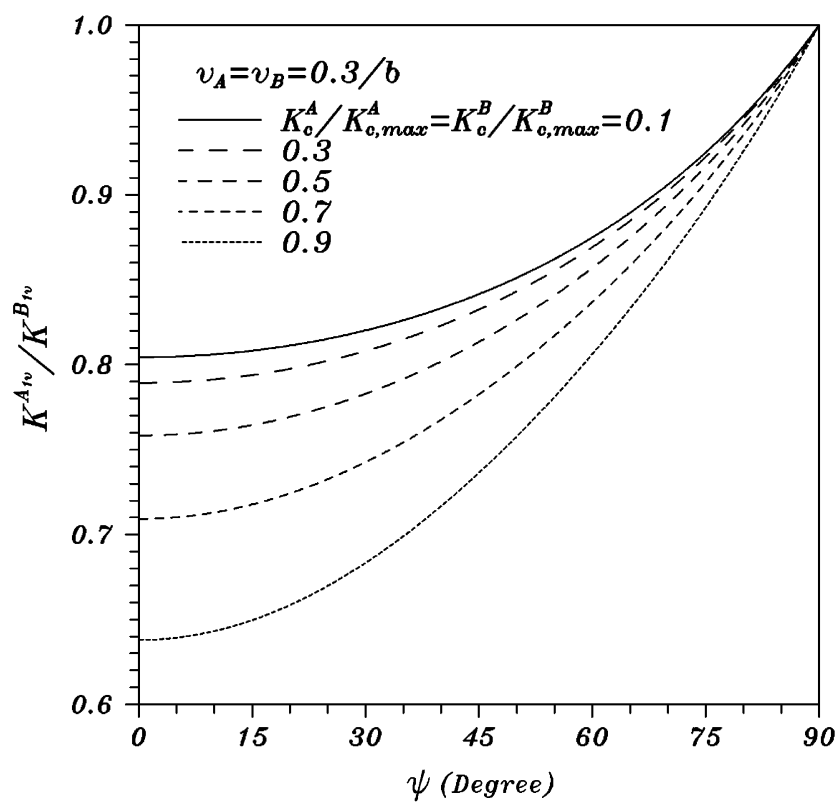

Fig. 14. The ratio of the stress intensity factor of propagating crack tips $A$ and $B$ for various fracture toughness.

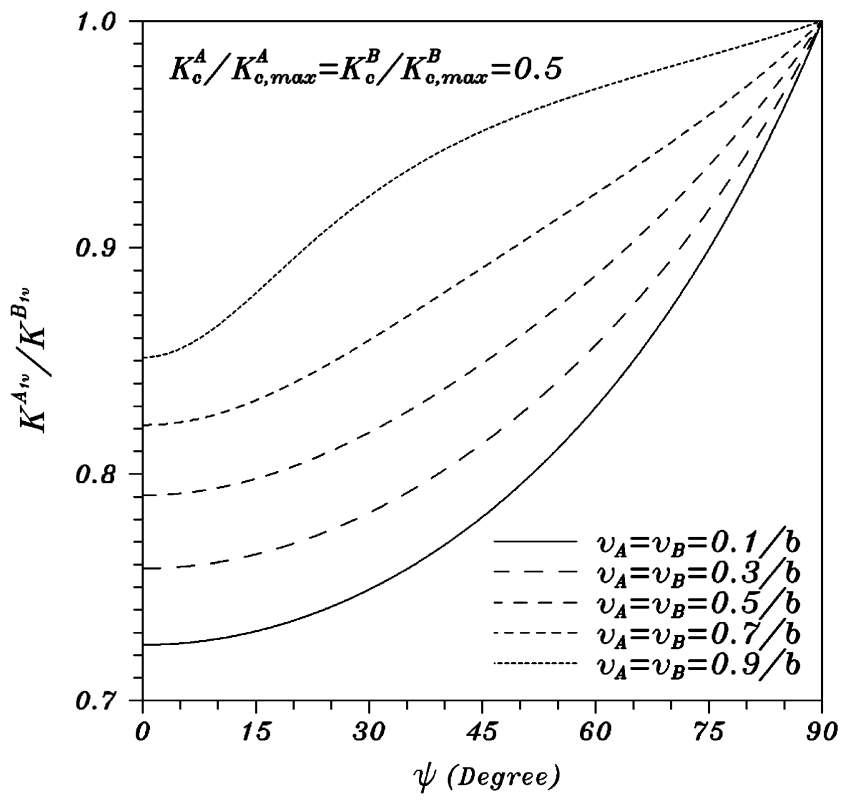

Fig. 15. The ratio of the stress intensity factor of propagating crack tips $A$ and $B$ for various crack speeds. 
propagating cracks. Multiple diffracted waves generated by two crack tips are taken into account. The exact transient solutions of dynamic stress intensity factors for stationary cracks are obtained in compact formulations which account for the contributions of all the diffracted waves. Each term in the formulations has its own physical meaning. The dynamic stress intensity factors for stationary cracks subjected to a step-stress wave increase with time and will reach a maximum value at the instance that the first diffracted wave generated from the other crack tip arrives at the tip. The ratio of the maximum value and the corresponding static value is $4 / \pi$ for both tips for various incident angles and material constants. The stress intensity factors decrease after the first diffracted wave has passed the crack tip and then oscillate near the static value. The stress intensity factor increases very rapidly in a short time period for the left crack tip $B$ so that the fracture tends to occur first at the crack tip $B$ for high fracture toughness.

For propagating crack, the two crack tips are assumed to propagate along the crack tip line with constant subsonic velocities as the stress intensity factor reaches the fracture toughness of the material. The influence of the first two diffracted waves generated from the other tip on the stress intensity factors of the propagating crack tip is investigated. It is interesting to note that the dynamic stress intensity factor during crack propagation of the crack tip $B$ is larger than that of the crack tip $A$, which is the tip that the incident plane wave first strikes. The diffracted waves have significant influence on the stress intensity factor of the cracks especially for the crack tip $B$. The influence of the diffracted waves on the dynamic stress intensity factor for higher propagation velocity is relatively smaller than for low velocity.

\section{Acknowledgements}

The authors gratefully acknowledge the financial support of this research by the National Science Council (Republic of China) under Grant NSC 88-2212-E-032-015.

\section{References}

Achenbach, J.D., 1970a. Brittle and ductile extension of a finite crack by a horizontally polarized shear wave. Int. J. Eng. Sci. 8, 947-966.

Achenbach, J.D., 1970b. Extension of a crack by a shear wave. Z. Math. Phys. (ZAMP) 21, 887-900.

Achenbach, J.D., 1973. Wave Propagation in Elastic Solids. North-Holland, Amsterdam.

Albuquerque, E.L., Sollero, P., Aliabadi, M.H., 2002. The boundary element method applied to time dependent problems in anisotropic materials. J. Solids Struct. 39, 1405-1422.

de Hoop, A.T., 1958. Representation theorems for the displacement in an elastic solid and their application to elastodynamic diffraction theory. Doctoral Dissertation, Technische Hogeschool, Delft.

Flitman, L.M., 1963. Waves generated by sudden crack in a continuous elastic medium. Appl. Math. Mech. (PMM) 27, 938-953.

Freund, L.B., 1972a. Crack propagation in an elastic solid subjected to general loading-I. Constant rate of extension. J. Mech. Phys. Solids 20, 129-140.

Freund, L.B., 1972b. Crack propagation in an elastic solid subjected to general loading-II. Non-uniform rate of extension. J. Mech. Phys. Solids 20, 141-152. 
Freund, L.B., 1973. Crack propagation in an elastic solid subjected to general loading-III. Stress wave loading. J. Mech. Phys. Solids 21, 47-61.

Freund, L.B., 1974a. The stress intensity factor due to normal impact loading of the faces of a crack. Int. J. Eng. Sci. 12, 179-189.

Freund, L.B., 1974b. Crack propagation in an elastic solid subjected to general loading-IV. Obliquely incident stress pulse. J. Mech. Phys. Solids 22, 137-146.

Freund, L.B., 1990. Dynamic Fracture Mechanics. Cambridge University Press, Cambridge.

Guo, F., Yang, W., Huang, Y., Rosakis, A.J., 2003. Sudden deceleration or acceleration of an intersonic shear crack. J. Mech. Phys. Solids 51, 311-331.

Ing, Y.S., Ma, C.C., 1996. Transient response of a finite crack subjected to dynamic anti-plane loading. Int. J. Fract. 82, 345-362.

Ing, Y.S., Ma, C.C., 1997. Dynamic fracture analysis of a finite crack subjected to an incident horizontally polarized shear wave. Int. J. Solids Struct. 34, 895-910.

Kassir, M.K., Bandyopadhyay, K.K., 1983. Impact response of a cracked orthotropic medium. J. Appl. Mech. 50, 630-636.

Kostrov, B.V., 1964. Self-similar problems of propagation of shear cracks. Appl. Math. Mech. (PMM) 28, $1077-1087$.

Kostrov, B.V., 1966. Unsteady propagation of longitudinal shear cracks. Prikl. Mat. Mekh. 30, 1241-1248.

Lin, R.L., Ma, C.C., 2000. Antiplane deformations for anisotropic multilayered media by using the coordinate transform method. J. Appl. Mech. 67, 597-605.

Ma, C.C., 1988. Initiation, propagation, and kinking of an in-plane crack. J. Appl. Mech. 55, 587-595.

Ma, C.C., 1990. Dynamic mixed mode I-II crack kinking under oblique stress wave loading in brittle solids. J. Appl. Mech. 57, 117-127.

Ma, C.C., 1996. Relationship of anisotropic and isotropic materials for antiplane problems. AIAA J. 34, 2453-2456.

Ma, C.C., Burgers, P., 1986. Mode III kinking with delay time: an analytical approximation. Int. J. Solids Struct. 22, 883-899.

Ma, C.C., Burgers, P., 1987. Dynamic mode I and mode II crack kinking including delay time effects. Int. J. Solids Struct. 23, 897-918.

Ma, C.C., Burgers, P., 1988. Initiation, propagation, and kinking of an antiplane crack. J. Appl. Mech. 55, 111-119.

Noble, B., 1958. Methods Based on the Wiener-Hopf Technique. Pergamon Press, Elmsford, NY.

Rosakis, A.J., 2002. Intersonic shear cracks and fault ruptures. Adv. Phys. 51, 1189-1257.

Rubio-Gonzalez, C., Mason, J.J., 1999. Response of finite cracks in orthotropic materials due to concentrated impact shear loads. J. Appl. Mech. 66, 485-491.

Rubio-Gonzalez, C., Mason, J.J., 2001. Green's functions for the stress intensity factor evolution in finite cracks in orthotropic materials. Int. J. Fract. 108, 317-335.

Samudrala, O., Rosakis, A.J., 2003. Effect of loading and geometry on the subsonic/intersonic transition of a bimaterial interface crack. Eng. Fract. Mech. 70, 309-337.

Shindo, Y., Nozaki, H., 1991. Impact response of a finite crack in an orthotropic strip. Acta Mech. 62, 87-104.

Sih, G.C., Embley, G.T., 1972. Impact response of a finite crack in plane extension. Int. J. Solids Struct. 8, 977-993.

Thau, S.A., Lu, T.H., 1971. Transient stress intensity factor for a finite crack in an elastic solid caused by a dilatational wave. Int. J. Solids Struct. 7, 731-750.

Tsai, C.H., Ma, C.C., 1992. Transient analysis of a semi-infinite crack subjected to dynamic concentrated forces. J. Appl. Mech. 59, 804-811.

Tsai, C.H., Ma, C.C., 1997a. Transient analysis of a propagating in-plane crack in a finite geometry body subjected to static loadings. J. Appl. Mech. 64, 620-628.

Tsai, C.H., Ma, C.C., 1997b. Theoretical transient analysis of the interaction between a dynamically propagating in-plane crack and traction free boundaries. J. Appl. Mech. 64, 819-827.

Yoffe, E.H., 1951. The moving Griffith crack. Philos. Mag. 42, 739-750.

Zhang, C., 2000. Transient elastodynamic antiplane crack analysis of anisotropic solids. Int. J. Solids Struct. 37, 6107-6130. 